Herbert Lange $\cdot$ Christian Pauly

\title{
Polarizations of Prym varieties for Weyl groups via abelianization
}

Received June 11, 2007 and in revised form September 29, 2007

\begin{abstract}
Let $\pi: Z \rightarrow X$ be a Galois covering of smooth projective curves with Galois group the Weyl group of a simple and simply connected Lie group $G$. For any dominant weight $\lambda$ consider the curve $Y=Z / \operatorname{Stab}(\lambda)$. The Kanev correspondence defines an abelian subvariety $P_{\lambda}$ of the Jacobian of $Y$. We compute the type of the polarization of the restriction of the canonical principal polarization of $\operatorname{Jac}(Y)$ to $P_{\lambda}$ in some cases. In particular, in the case of the group $E_{8}$ we obtain families of Prym-Tyurin varieties. The main idea is the use of an abelianization map of the DonagiPrym variety to the moduli stack of principal $G$-bundles on the curve $X$.
\end{abstract}

Keywords. Prym variety, principal G-bundle, abelianization, moduli stack

\section{Introduction}

\subsection{Verlinde spaces}

Let $X$ be a smooth complex projective curve of genus $g$ and let $G$ be a simple, simply connected complex Lie group. We denote by $\mathcal{M}_{X}(G)$ the moduli stack of principal $G$-bundles and by $\mathcal{L}$ the ample generator of its Picard group. The celebrated Verlinde formula ([Fa1], [So1], [So2] $)$ gives the dimension $N_{g, l}(G)$ of the space of global sections $H^{0}\left(\mathcal{M}_{X}(G), \mathcal{L}^{\otimes l}\right)$ for any level $l$. The Verlinde numbers at level $l=1$ for the groups of type $\mathrm{ADE}$ are given in the following table.

\begin{tabular}{c|ccccc}
\hline$G$ & $\mathrm{SL}(m)$ & $\operatorname{Spin}(2 m)$ & $E_{6}$ & $E_{7}$ & $E_{8}$ \\
$N_{g, 1}(G)$ & $m^{g}$ & $4^{g}$ & $3^{g}$ & $2^{g}$ & 1 \\
\hline
\end{tabular}

The number $m^{g}$ for $\operatorname{SL}(m)$ coincides with the number of level- $m$ theta functions on the Jacobian of $X$ (see $[\overline{\mathrm{BNR}}]$ ). For the even Spin group the Verlinde number equals the number of theta characteristics of $X$ (see [O] $)$. The striking simplicity of the Verlinde

H. Lange; Mathematisches Institut, Universität Erlangen-Nürnberg, Bismarckstrasse 1 1/2, D-91054 Erlangen, Deutschland; e-mail: lange@mi.uni-erlangen.de

C. Pauly: Département de Mathématiques, Université de Montpellier II, Case Courrier 051, Place Eugène Bataillon, 34095 Montpellier Cedex 5, France; e-mail: pauly@math.univ-montp2.fr

Mathematics Subject Classification (2000): Primary 14H40, 14K25, 14H30; Secondary 14H60, 14D20 
numbers for $E_{6}, E_{7}$ and $E_{8}$ was the main motivation for us to try to relate these Verlinde spaces to spaces of theta functions on polarized abelian varieties (the Prym varieties) and compute the induced polarizations—-see Main Theorem and Remark 8.4 .

\subsection{Abelianization of principal $G$-bundles}

The abelianization program of principal $G$-bundles, or more precisely $G$-Higgs bundles, takes its origin in Hitchin's papers [Hi1] and [Hi2]. For the case $G=\mathrm{SL}(m)$ it is shown in [BNR] that for a sufficiently ramified spectral cover $\psi: Y \rightarrow X$ the direct image map

$$
\operatorname{Prym}(Y / X) \rightarrow \mathcal{M}_{X}(\operatorname{SL}(m))
$$

induces by pull-back an isomorphism between the SL( $m)$-Verlinde space at level 1 and the space of abelian theta functions $H^{0}\left(\operatorname{Prym}(Y / X), L_{Y}\right)$. For general structure groups $G$ the abelianization theory has been worked out by Faltings [Fa2], Donagi [Don1], [Don2] and Donagi-Gaitsgory [DG].

\subsection{Correspondences on spectral and cameral covers}

For general structure groups $G$ Prym varieties can be constructed via correspondences on covers of the curve $X$ :

In [K1] Kanev constructs from the data of a rational map $f: \mathbb{C} \rightarrow \mathfrak{g}=\operatorname{Lie}(G)$ and an irreducible representation $\rho_{\lambda}: G \rightarrow \operatorname{GL}(V)$ a spectral cover $\psi: Y \rightarrow \mathbb{P}^{1}$ equipped with a correspondence. He shows that if $G$ is of type ADE, the weight $\lambda$ minuscule and $f$ sufficiently general, then the Prym variety $P_{\lambda} \subset \operatorname{Jac}(Y)$ associated with Kanev's correspondence is a Prym-Tyurin variety (i.e. the polarization on $P_{\lambda}$ induced from the principal polarization of $\operatorname{Jac}(Y)$ is a multiple of a principal polarization). Note that Kanev's construction is carried out in the case $X=\mathbb{P}^{1}$.

A different but related construction of Prym varieties is given by Donagi in [Don1], [Don2]. Let $T \subset G$ be a maximal torus, $W$ the Weyl group of $G$ and $\mathbb{S}_{\omega}=\operatorname{Hom}\left(T, \mathbb{C}^{*}\right)$ the weight lattice. For any cameral cover, i.e. a Galois cover $\pi: Z \rightarrow X$ with Galois group $W$, satisfying some conditions on the ramification, Donagi introduces the Prym variety $\operatorname{Prym}\left(\pi, \mathbb{S}_{\omega}\right):=\operatorname{Hom}_{W}\left(\mathbb{S}_{\omega}, \operatorname{Jac}(Z)\right)$ parametrizing $W$-equivariant homomorphisms from $\mathbb{S}_{\omega}$ to $\operatorname{Jac}(Z)$.

In this paper we generalize Kanev's construction to an arbitrary base curve $X$. Given a Galois cover $\pi: Z \rightarrow X$ and a dominant weight $\lambda \in \mathbb{S}_{\omega}$ we consider the cover of curves $\psi: Y \rightarrow X$ with $Y=Z / \operatorname{Stab}(\lambda)$. Kanev's construction generalizes (see Section 3) to give a correspondence $\bar{K}_{\lambda}$ on the curve $Y$ defining an abelian subvariety $P_{\lambda} \subset \operatorname{Jac}(Y)$, which is isogenous to the Donagi-Prym variety $\operatorname{Prym}\left(\pi, \mathbb{S}_{\omega}\right)$ (Proposition 6.13).

\subsection{The polarization on the Prym variety $P_{\lambda}$}

Let $L_{Y}$ denote a line bundle defining the canonical principal polarization on the Jacobian $\operatorname{Jac}(Y)$. The aim of this paper is to compute the induced polarization $L_{Y} \mid P_{\lambda}$ under certain 
assumptions. In fact, if $q_{\lambda}$ denotes the exponent of the correspondence $\bar{K}_{\lambda}$ and $d_{\lambda}$ the Dynkin index of $\lambda$, our main result is the following theorem (we prove a slightly more precise version, see Theorem 8.1]. We use the notation of [B] for the weights.

Main Theorem. Suppose that the $W$-Galois cover $\pi: Z \rightarrow X$ is étale. Then in the cases given in the table below the induced polarization $L_{Y} \mid P_{\lambda}$ is divisible by $q_{\lambda}$, i.e. $L_{Y} \mid P_{\lambda}=M^{\otimes q_{\lambda}}$ and the polarization $M$ on $P_{\lambda}$ is of type $K(M)=(\mathbb{Z} / m \mathbb{Z})^{2 g}$ :

\begin{tabular}{cccc}
\hline Weyl group of type & weight $\lambda$ & $q_{\lambda}=d_{\lambda}$ & $K(M)$ \\
$A_{n}, n>1$ & $\varpi_{i},(i, n+1)=1$ & $\left(\begin{array}{c}n-1 \\
i-1\end{array}\right)$ & $(\mathbb{Z} /(n+1) \mathbb{Z})^{2 g}$ \\
$D_{n}, n$ odd & $\varpi_{n-1}, \varpi_{n}$ & $2^{n-3}$ & $(\mathbb{Z} / 4 \mathbb{Z})^{2 g}$ \\
$E_{6}$ & $\varpi_{1}, \varpi_{6}$ & 6 & $(\mathbb{Z} / 3 \mathbb{Z})^{2 g}$ \\
$E_{7}$ & $\varpi_{7}$ & 12 & $(\mathbb{Z} / 2 \mathbb{Z})^{2 g}$ \\
$E_{8}$ & $\varpi_{8}$ & 60 & 0 \\
\hline
\end{tabular}

Using the table in Section 1.1 we observe that in all the cases of the Main Theorem we have an equality of dimensions

$$
\operatorname{dim} H^{0}\left(P_{\lambda}, M\right)=N_{g, 1}(G) .
$$

Moreover, there exists a morphism $\gamma: P_{\lambda} \rightarrow \mathcal{M}_{X}(G)$ (see Remark 8.3) constructed via the abelianization map $\Delta_{\theta}$ (see below) and which induces by pull-back a linear map between spaces of global sections having the same dimension

$$
\gamma^{*}: H^{0}\left(\mathcal{M}_{X}(G), \mathcal{L}\right) \rightarrow H^{0}\left(P_{\lambda}, M\right) .
$$

We discuss in Remark 8.4 the natural question whether $\gamma^{*}$ is an isomorphism.

It is well known that the Weyl group of type $E_{k}$ is closely related to the del Pezzo surface of degree $9-k$ for $5 \leq k \leq 8$. In fact, a slightly modified lattice of the weight lattice is isomorphic to the Picard lattice of the corresponding del Pezzo surface (see [K1] Section 8.7]). Moreover, for $4 \leq k \leq 7$ the Kanev correspondence is given essentially by the incidence correspondence of lines of the corresponding del Pezzo surface. For $k=8$ there are multiplicities due to the fact that the weight $\varpi_{8}$ is only quasi-minuscule (see [K1]). Notice that in these cases the polarization $M$ on $P_{\lambda}$ is of type $(\mathbb{Z} / d \mathbb{Z})^{2 g}$ where $d$ is the degree of the corresponding del Pezzo surface.

In particular, in the case of $W=W\left(E_{8}\right)$ we obtain a family of Prym-Tyurin varieties, i.e. the pairs $\left(P_{\lambda}, M\right)$ are principally polarized abelian varieties. It is easy to see that any curve $X$ of genus $g \geq 4$ admits an étale Galois covering with Galois group $W\left(E_{8}\right)$ and we get a family of Prym-Tyurin varieties of dimension $8(g-1)$ of exponent 60. We plan to study this family in a subsequent paper.

Probably there is an analogous result in the case where the Galois covering $\pi$ : $Z \rightarrow X$ admits simple ramification. Certainly the paper [DG] will be essential for this. We plan to come back to this subsequently.

Note that our results are disjoint from the results in [K1]. First of all, Kanev considers only Galois coverings over $\mathbb{P}^{1}$ which are necessarily ramified. Moreover, his corre- 
spondence satisfies a quadratic equation and he uses his criterion [K2] to show that the associated abelian subvarieties are principally polarized. In our case the relevant correspondence satisfies a cubic equation (see Theorem 3.9p and a quadratic equation only on the Prym variety $\operatorname{Prym}(Y / X)$ of the covering $\psi: Y \rightarrow X$. However, the abelian variety $\operatorname{Prym}(Y / X)$ is not principally polarized and thus we cannot apply Kanev's criterion [K2] in order to compute the polarization of $P_{\lambda}$.

Instead we proceed as follows: We work out a general result on restrictions of polarizations to abelian subvarieties (Proposition 2.10) roughly saying that, if the restricted polarization equals the $q$-fold of a polarization, where $q$ is the exponent of the abelian subvariety, then its type can be computed.

The main idea of the proof is then to use an abelianization map

$$
\Delta_{\theta}: \operatorname{Prym}\left(\pi, \mathbb{S}_{\omega}\right)_{n} \rightarrow \mathcal{M}_{X}(G)
$$

(see Section 7). Here $\operatorname{Prym}\left(\pi, \mathbb{S}_{\omega}\right)_{n}$ denotes a certain connected component of the Donagi-Prym variety $\operatorname{Prym}\left(\pi, \mathbb{S}_{\omega}\right)$. The fact that the restricted polarization is the $q_{\lambda}$-fold of a polarization is a consequence of the existence of a commutative diagram

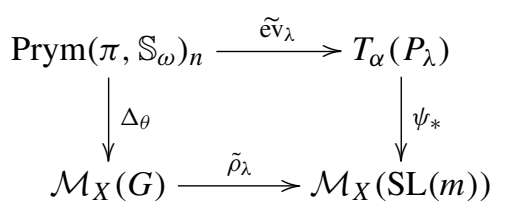

(here $\tilde{\mathrm{ev}}_{\lambda}$ denotes evaluation at $\lambda$, the map $\tilde{\rho}_{\lambda}$ is induced by the representation with dominant weight $\lambda$, and $T_{\alpha}$ is a certain translation on $\left.\operatorname{Jac}(Y)\right)$ together with a theorem of Laszlo-Sorger ([LS], [So2]) saying that the pull-back of the determinant bundle on $\mathcal{M}_{X}(\operatorname{SL}(m))$ by $\tilde{\rho}_{\lambda}$ is the $d_{\lambda}$-fold of the ample generator of $\operatorname{Pic}\left(\mathcal{M}_{X}(G)\right)$, and the assumption $q_{\lambda}=d_{\lambda}$. Note that in all the cases of the Main Theorem $\operatorname{Prym}\left(\pi, \mathbb{S}_{\omega}\right)_{n}$ and $P_{\lambda}$ are isomorphic.

The contents of the paper are as follows: In Section 2 we prove the result just mentioned concerning restriction of a polarization to an abelian subvariety. In Section 3 we prove that the Schur and the Kanev correspondences belong to the same abelian subvariety. This was shown in [LRo] for the special case $X=\mathbb{P}^{1}$ and for a left action of the group. In Section 4 we compute the invariants of our main examples mentioned above. In Section 5 we introduce the Donagi-Prym variety and derive the properties we need. Section 6 contains some results on Mumford groups. In Section 7 we introduce the abelianization map and prove the commutativity of the above diagram. Section 8 contains the proof of our main theorem.

Finally, a word on the group actions: The group $W$ acts on the curve $Z$ as well as on the weight lattice $\mathbb{S}_{\omega}$ and these actions have to be consistently either both left actions or both right actions. Since traditionally principal bundles are defined via right actions, we are forced to use right actions in both cases. 


\section{Restriction of polarizations to abelian subvarieties}

Let $S$ be an abelian variety with polarization $L$ and associated isogeny $\varphi_{L}: S \rightarrow \hat{S}$. Define as usual $K(L)=\operatorname{ker} \varphi_{L}$. We consider an endomorphism $u \in \operatorname{End}(S)$ with the following properties:

- $u$ is symmetric with respect to the Rosati involution, i.e.,

$$
\varphi_{L} u=\hat{u} \varphi_{L},
$$

where $\hat{u} \in \operatorname{End}(\hat{S})$ is the dual endomorphism.

- There exists a positive integer $q$ such that

$$
u^{2}=q u \text {. }
$$

We introduce the abelian subvarieties of $S$

$$
A=\operatorname{im} u \subset \operatorname{ker}(q-u) \quad \text { and } \quad P=\operatorname{im}(q-u) \subset \operatorname{ker} u
$$

and we wish to study the induced polarizations $L_{A}=L \mid A$ on $A$ and $L_{P}=L \mid P$ on $P$, i.e. determine the subgroups $K\left(L_{P}\right)$ and $K\left(L_{A}\right)$.

We use the following notation:

$$
\iota_{A}: A \hookrightarrow S, \quad \iota_{P}: P \hookrightarrow S, \quad \pi_{A}: S \rightarrow S / A, \quad \pi_{P}: S \rightarrow S / P,
$$

where the $\iota$ 's are inclusions and $\pi$ 's are projections.

Remark 2.1. (i) For any abelian subvariety $A$ of $S$ there is an endomorphism $u$ satisfying (1) and (2). In fact, we can take the composition

$$
u=\iota_{A} \psi_{L_{A}} \hat{\iota}_{A} \varphi_{L} \in \operatorname{End}(S)
$$

(see [BL, Lemma 5.3.1]). Here $\psi_{L_{A}}=q \varphi_{L}^{-1}: \hat{A} \rightarrow A$ is an isogeny.

(ii) Let $\psi: Y \rightarrow X$ be a degree $d$ cover of smooth projective curves. If we identify the Jacobians $\operatorname{Jac}(X)$ and $\operatorname{Jac}(Y)$ with their duals via the canonical polarizations, then the dual $\widehat{\psi^{*}}$ of the map $\psi^{*}: \operatorname{Jac}(X) \rightarrow \operatorname{Jac}(Y)$ coincides with the norm map $\mathrm{Nm}: \operatorname{Jac}(Y) \rightarrow \operatorname{Jac}(X)$. Moreover, the endomorphism

$$
t=\psi^{*} \mathrm{Nm} \in \operatorname{End}(\operatorname{Jac}(Y))
$$

satisfies (1) and (2) with $q=d$. The abelian subvariety $P=\operatorname{im}(d-t)$ defined in (3) coincides with the usual Prym variety (not necessarily principally polarized) of the morphism $\psi$, which we denote by $\operatorname{Prym}(Y / X)$, i.e.,

$$
P=\operatorname{Prym}(Y / X)=(\operatorname{kerNm})_{0} .
$$

The endomorphism $t$ is induced by the trace correspondence $\bar{T}$ on $Y$ (see Section 3). We denote the kernel of $\psi^{*}: \operatorname{Jac}(X) \rightarrow \operatorname{Jac}(Y)$ by $K$. We also have the equality

$$
\operatorname{im} \psi^{*}=\operatorname{Jac}(X) / K=\operatorname{im} t .
$$

Since the norm map $\mathrm{Nm}$ is the dual of $\psi$ we easily see that the group of connected components of the fibre $\mathrm{Nm}^{-1}(0)$ is isomorphic to $K$. 
(iii) With $u$ also $u^{\prime}=q-u$ satisfies (1) and (2). Replacing $u$ by $u^{\prime}$ interchanges the abelian subvarieties $A$ and $P$. Hence for the following lemmas it suffices to prove one of the two statements concerning $A$ and $P$, and we will do so without further mention.

Recall that the addition map $\mu$ of $S$ satisfies $\mu=\iota_{A}+\iota_{P}$ and the following sequence is exact:

$$
0 \rightarrow A \cap P \rightarrow A \times P \stackrel{\mu}{\longrightarrow} S \rightarrow 0 .
$$

For any abelian variety $B$ and any positive integer $n$ let $B_{n}$ denote the subgroup of $n$ torsion points of $B$. We denote by $|M|$ the cardinality of a set $M$. With this notation we have the following lemmas.

Lemma 2.2. The finite subgroup $A \cap P$ is contained in $A_{q}$ and $P_{q}$.

Proof. Let $x \in A \cap P$. Then $u(x)=q x$, since $x \in A$, and $u(x)=0$, since $x \in P$. This implies the assertion.

Lemma 2.3. $\left|K\left(L_{A}\right)\right| \cdot\left|K\left(L_{P}\right)\right|=|A \cap P|^{2} \cdot|K(L)|$.

Proof. According to [BL, Corollary 5.3.6] the polarization $\mu^{*}(L)$ splits, i.e. $\varphi_{\mu^{*}(L)}=$ $\varphi_{L_{A}} \times \varphi_{L_{P}}$, which gives

$$
\left|K\left(\mu^{*} L\right)\right|=\left|K\left(L_{A}\right)\right| \cdot\left|K\left(L_{P}\right)\right| .
$$

On the other hand, (4) implies $\operatorname{deg}(\mu)=|A \cap P|$ and thus

$$
\left|K\left(\mu^{*} L\right)\right|=\left|\operatorname{ker} \mu \varphi_{L} \hat{\mu}\right|=\operatorname{deg}(\mu)^{2} \cdot\left|\operatorname{ker} \varphi_{L}\right|=|A \cap P|^{2} \cdot|K(L)| .
$$

Combining both equations gives the assertion.

Lemma 2.4. We have the following equalities of abelian subvarieties of $\hat{S}$ :

$$
\varphi_{L}(P)=\widehat{S / A} \subset \hat{S} \text { and } \varphi_{L}(A)=\widehat{S / P} \subset \hat{S} .
$$

Proof. The endomorphism $u$ factorizes as $i_{A} v$ with $v: S \rightarrow A$. Taking the dual gives the factorization

$$
\hat{u}: \hat{S} \stackrel{\hat{\iota}_{A}}{\longrightarrow} \hat{A} \stackrel{\hat{v}}{\longrightarrow} \hat{S},
$$

from which we deduce that $\operatorname{ker} \hat{\iota}_{A}=\widehat{S / A} \subset \operatorname{ker} \hat{u}$. On the other hand, we have $P \subset$ ker $u$ and so (1) implies $\varphi_{L}(P) \subset \operatorname{ker} \hat{u}$. This gives the first equality, since both abelian subvarieties are the connected component of the origin of ker $\hat{u}$, as they are of the same dimension.

We will give two descriptions of the subgroups $K\left(L_{P}\right)\left(=\operatorname{ker}\left(\varphi_{L_{P}}: P \stackrel{\iota_{P}}{\longrightarrow} S \stackrel{\varphi_{L}}{\longrightarrow} \hat{S}\right.\right.$ $\left.\stackrel{\hat{\iota}_{P}}{\longrightarrow} \hat{P}\right)$ ) and $K\left(L_{A}\right)$. We observe that $\operatorname{ker} \hat{\iota}_{P}=\widehat{S / P}$ and, according to Lemma 2.4 $\widehat{S / P}=\varphi_{L}(A)$. Hence we obtain

$$
K\left(L_{P}\right)=\bigcup_{x \in K(L)}(A+x) \cap P,
$$


where $A+x$ denotes the image of $A$ under translation by $x$. Similarly we have

$$
K\left(L_{A}\right)=\bigcup_{x \in K(L)}(P+x) \cap A .
$$

In particular, $A \cap P \subset K\left(L_{P}\right)$ and $A \cap P \subset K\left(L_{A}\right)$. For the second description consider the isogenies

$$
\alpha=\pi_{A} \iota_{P}: P \stackrel{\iota_{P}}{\longrightarrow} S \stackrel{\pi_{A}}{\longrightarrow} S / A \quad \text { with } \quad \operatorname{ker} \alpha=A \cap P
$$

and

$$
\beta=\pi_{P} \iota_{A}: A \stackrel{\iota_{A}}{\longrightarrow} S \stackrel{\pi_{P}}{\longrightarrow} S / P \quad \text { with } \quad \operatorname{ker} \beta=A \cap P .
$$

Moreover, define the isogenies

$$
\varphi_{P}: P \rightarrow \widehat{S / A} \text { and } \varphi_{A}: A \rightarrow \widehat{S / P}
$$

by the factorizations $\varphi_{L} \mid P: P \stackrel{\varphi_{P}}{\longrightarrow} \widehat{S / A} \hookrightarrow \hat{S}$ and $\varphi_{L} \mid A: A \stackrel{\varphi_{A}}{\longrightarrow} \widehat{S / P} \hookrightarrow \hat{S}$ of Lemma 2.4. With this notation we have

Lemma 2.5. The following sequences are exact:

$$
\begin{aligned}
& 0 \rightarrow A \cap P \hookrightarrow K\left(L_{P}\right) \stackrel{\alpha}{\longrightarrow} \operatorname{ker} \hat{\varphi}_{P} \rightarrow 0 \quad \text { with } \quad K(L) \cap P \cong \operatorname{ker} \hat{\varphi}_{P} \subset S / A, \\
& 0 \rightarrow A \cap P \hookrightarrow K\left(L_{A}\right) \stackrel{\beta}{\longrightarrow} \operatorname{ker} \hat{\varphi}_{A} \rightarrow 0 \quad \text { with } \quad K(L) \cap A \cong \operatorname{ker} \hat{\varphi}_{A} \subset S / P .
\end{aligned}
$$

Proof. The dual isogeny of $\alpha$ factorizes as

$$
\hat{\alpha}: \widehat{S / A} \stackrel{\hat{\pi}_{A}}{\longrightarrow} \hat{S} \stackrel{\hat{\iota}_{P}}{\longrightarrow} \hat{P} .
$$

By the previous lemma $\varphi_{L}(P)=\widehat{S / A}$, which implies that

$$
\varphi_{L_{P}}=\hat{\iota}_{P} \varphi_{L} \iota_{P}=\hat{\alpha} \varphi_{L} \iota_{P} .
$$

Hence $\varphi_{L_{P}}$ factorizes as $\varphi_{L_{P}}: P \stackrel{\varphi_{P}}{\longrightarrow} \widehat{S / A} \stackrel{\hat{\alpha}}{\longrightarrow} \hat{P}$. Taking the dual and using $\hat{\varphi}_{L_{P}}=\varphi_{L_{P}}$ we obtain the factorization

$$
\varphi_{L_{P}}: P \stackrel{\alpha}{\longrightarrow} S / A \stackrel{\hat{\varphi}_{P}}{\longrightarrow} \hat{P}
$$

which gives the exact sequence $(5)$. The assertion on the kernel follows from $\operatorname{ker}\left(\hat{\varphi}_{P}\right) \cong$ $\operatorname{ker}\left(\varphi_{P}\right)=K(L) \cap P$.

In particular, we obtain

$$
\left|K\left(L_{P}\right)\right|=|A \cap P| \cdot|K(L) \cap P| \quad \text { and } \quad\left|K\left(L_{A}\right)\right|=|A \cap P| \cdot|K(L) \cap A| .
$$

Inserting this into Lemma 2.3 yields

Corollary 2.6. $|K(L) \cap A| \cdot|K(L) \cap P|=|K(L)|$. 
Consider the finite groups

$$
G_{P}=\operatorname{ker} u / P \quad \text { and } \quad G_{A}=\operatorname{ker}(q-u) / A .
$$

They are the groups of connected components of $\operatorname{ker} u$ and $\operatorname{ker}(q-u)$ respectively.

Lemma 2.7. There are canonical exact sequences

$$
0 \rightarrow A \cap P \rightarrow A_{q} \rightarrow G_{P} \rightarrow 0 \text { and } 0 \rightarrow A \cap P \rightarrow P_{q} \rightarrow G_{A} \rightarrow 0 .
$$

Proof. Since $P \subset \operatorname{ker} u$ the endomorphism $u$ descends to an isogeny $\bar{u}: S / P \rightarrow A$ with $\operatorname{ker} \bar{u}=G_{P}$. Moreover, we know that $u \mid A=q$, which implies that the composite isogeny $A \stackrel{\bar{\pi}_{P \iota_{A}}}{\longrightarrow} S / P \stackrel{\bar{u}}{\longrightarrow} A$ equals $q$. Taking kernels leads to the first exact sequence of the lemma.

We denote by

$$
u_{q}: S_{q} \rightarrow A_{q} \quad \text { and } \quad(q-u)_{q}: S_{q} \rightarrow P_{q}
$$

the restrictions of $u$ and $q-u$ to the $q$-torsion points $S_{q}$. Note that $u_{q}$ and $(q-u)_{q}$ are nilpotent: $u_{q}^{2}=(q-u)_{q}^{2}=0$. Note moreover that $G_{P} \subset(S / P)_{q}$ and $G_{A} \subset(S / A)_{q}$.

Lemma 2.8. We have the following equalities of finite groups:

$\operatorname{im} u_{q}=A \cap P, \quad \operatorname{coker} u_{q}=G_{P}, \quad \operatorname{im}(q-u)_{q}=A \cap P, \quad \operatorname{coker}(q-u)_{q}=G_{A}$.

Proof. First we claim that $\operatorname{im} u_{q} \subset A \cap P$. For the proof let $a \in \operatorname{im} u_{q}$. Write $a=u(s)$ with $s \in S_{q}$. Then $q s=0$ and therefore $a=(u-q)(s) \in P$.

In order to show equality of these subgroups of $A_{q}$ we compute their indices. According to Lemma 2.7 the index of $A \cap P$ in $A_{q}$ is $\left|G_{P}\right|$. The index of im $u_{q}$ in $A_{q}$ equals

$$
\frac{\left|A_{q}\right|}{\left|\operatorname{im} u_{q}\right|}=\frac{\left|A_{q}\right|}{\left|S_{q}\right|} \cdot\left|\operatorname{ker} u_{q}\right|=\frac{\left|\operatorname{ker} u_{q}\right|}{\left|P_{q}\right|} .
$$

Moreover, since $G_{P} \subset(S / P)_{q}$ the exact sequence $0 \rightarrow P \rightarrow \operatorname{ker} u \rightarrow G_{P} \rightarrow 0$ remains exact after taking $q$-torsion points. This implies the first equality. The second equality is obvious.

Consider again the isogenies $\hat{\varphi}_{P}: S / A \rightarrow \hat{P}$ and $\hat{\varphi}_{A}: S / P \rightarrow \hat{A}$.

Lemma 2.9. We have the following equalities of subgroups of $S / A$ and $S / P$ respectively:

$$
\operatorname{ker} \hat{\varphi}_{P}=\pi_{A}(K(L)) \quad \text { and } \operatorname{ker} \hat{\varphi}_{A}=\pi_{P}(K(L)) .
$$

Proof. The inclusion $\pi_{A}(K(L)) \subset \operatorname{ker} \hat{\varphi}_{P}$ follows from the commutative diagram

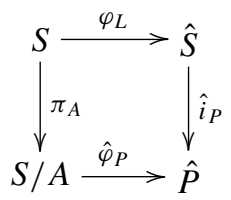


So it will be enough to show that these two groups have the same order. We have, using (5),

$$
\left|\operatorname{ker} \hat{\varphi}_{P}\right|=|K(L) \cap P| \quad \text { and } \quad\left|\pi_{A}(K(L))\right|=\frac{|K(L)|}{|K(L) \cap A|} .
$$

Equality follows from Corollary 2.6

The following proposition will be applied in the proof of the main theorem (Section 8).

Proposition 2.10. (a) If $L_{A}=M^{q}$ for a polarization $M$ on $A$, we have the following equality of subgroups of $A$ :

$$
K(M)=u(K(L)),
$$

(b) If $L_{P}=N^{q}$ for a polarization $N$ on $P$, we have the following equality of subgroups of $P$ :

$$
K(N)=(q-u)(K(L))
$$

Note that $L_{A}=M^{q}$ for a polarization $M$ on $A$ if and only if the polarization $L_{A}$ is divisible by $q$, meaning that $A_{q} \subset K\left(L_{A}\right)$. In this case $K(M)=K\left(L_{A}\right) / A_{q}$.

Proof. We take the quotient by $A \cap P$ of the inclusion $A_{q} \subset K\left(L_{A}\right)$. Since by Lemma 2.7. $A_{q} / A \cap P \cong G_{P}$, and by Lemma 2.5. $K\left(L_{A}\right) / A \cap P \cong \operatorname{ker} \hat{\varphi}_{A}$, this gives an inclusion

$$
G_{P} \subset \operatorname{ker} \hat{\varphi}_{A} \text {. }
$$

Recall that $G_{P}=\operatorname{ker} u / P$ and by Lemma 2.9 , $\operatorname{ker} \hat{\varphi}_{A}=\pi_{P}(K(L))$. Therefore

$$
\begin{aligned}
K(M)=K\left(L_{A}\right) / A_{q}=\operatorname{ker} \hat{\varphi}_{A} / G_{P} & =((K(L)+P) / P) /(\operatorname{ker} u / P) \\
& =(K(L)+P) / \operatorname{ker} u=u(K(L)) .
\end{aligned}
$$

\section{The Schur and Kanev correspondences}

\subsection{Representations of the Weyl group $W$}

Let $W$ be a Weyl group and let $\widehat{W}$ denote the set of its irreducible characters. It is known (see e.g. [Sp, Corollary 1.15]) that all irreducible representations of $W$ are defined over $\mathbb{Q}$. Therefore any irreducible representation of $W$ is also absolutely irreducible. Given $\omega \in \widehat{W}$ we choose an irreducible $\mathbb{Z}[W]$-module $\mathbb{S}_{\omega}$ such that

$$
V_{\omega}:=\mathbb{S}_{\omega} \otimes_{\mathbb{Z}} \mathbb{Q}
$$

is the irreducible representation of $W$ corresponding to $\omega$. As outlined in the introduction, we consider every representation of $W$ as a right representation. In particular, $\mathbb{S}_{\omega}$ is a right $\mathbb{Z}[W]$-module with action $(\lambda, g) \mapsto \lambda g$ for $\lambda \in \mathbb{S}_{\omega}$ and $g \in W$.

Fix a weight $\lambda$ of the lattice $\mathbb{S}_{\omega}$, which is by definition (see [K1, p. 158]) a vector

$$
\lambda \in V_{\omega} \quad \text { such that } \quad \lambda g-\lambda \in \mathbb{S}_{\omega} \forall g \in W .
$$


Let $\pi: Z \rightarrow X$ denote a Galois covering of smooth projective curves with Galois group $W$. We consider the action of $W$ on $Z$ as a right action $(z, g) \mapsto z^{g}$ for $z \in Z$ and $g \in W$. Let $H:=\operatorname{Stab}(\lambda) \subset W$ denote the stabilizer subgroup of the weight $\lambda \in V_{\omega}$. Then $\pi$ factorizes as

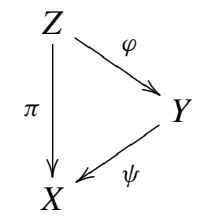

where $Y$ denotes the quotient $Z / H$. Schur's orthogonality relations induce a correspondence on $Z$ which we denote by $S_{\lambda}$. On the other hand, Kanev [K1] defined in the case $X=\mathbb{P}^{1}$ a correspondence on the curve $Y$, which we denote by $\bar{K}_{\lambda}$. It is the aim of this section to generalize Kanev's construction to an arbitrary base curve $X$ and to work out the relation between the two correspondences $S_{\lambda}$ and $\bar{K}_{\lambda}$.

\subsection{Schur correspondence}

Since $V_{\omega}$ is an absolutely irreducible $W$-representation, there is a unique negative definite $W$-invariant symmetric form ( , ) on $V_{\omega}$ such that

(1) $(\lambda, \mu) \in \mathbb{Z}$ for all $\mu \in \mathbb{S}_{\omega}$,

(2) any $W$-invariant form on $V_{\omega}$ satisfying (1) is an integer multiple of ( , ).

The Schur correspondence associated to the pair $\left(\mathbb{S}_{\omega}, \lambda\right)$ is by definition the rational correspondence on $Z$ over $X$ defined by

$$
S_{\lambda}=\sum_{g \in W}(\lambda g, \lambda) \Gamma_{g}
$$

Here $\Gamma_{g} \in Z \times Z$ denotes the graph of the automorphism $g$ of $Z$. Note that $(\lambda g, \lambda)$ need not be an integer. Considered as a map of $Z$ into the group $\operatorname{Div}_{\mathbb{Q}}(Z)$ of rational divisors on $Z, S_{\lambda}$ is given by

$$
S_{\lambda}(z)=\sum_{g \in W}(\lambda g, \lambda) z^{g}
$$

The correspondence $S_{\lambda}$ descends to a correspondence $\bar{S}_{\lambda}$ on $Y$ in the usual way,

$$
\bar{S}_{\lambda}=(\varphi \times \varphi)_{*} S_{\lambda} \subset Y \times Y .
$$

In order to express this correspondence as a map $Y \rightarrow \operatorname{Div}_{\mathbb{Q}}(Y)$, define $d=[W: H]$ and let $\left\{g_{1}=1, g_{2}, \ldots, g_{d}\right\}$ denote a set of representatives for the right cosets of $H$ in $W$, i.e. $W=\bigcup_{i=1}^{d} H g_{i}$.

Proposition 3.1. For any $y=\varphi(z) \in Y$ we have

$$
\bar{S}_{\lambda}(y)=|H|^{2} \sum_{i=1}^{d}\left(\lambda g_{i}, \lambda\right) \varphi\left(z^{g_{i}^{-1}}\right) .
$$


Note that the left-hand side of this equality depends neither on the choice of the point $z$ in the fibre over $y$ nor on the set of representatives $\left\{g_{i}\right\}$.

Proof. Since $H$ is the stabilizer of $\lambda$, by definition of $\bar{S}_{\lambda}$ we have

$$
\begin{aligned}
\bar{S}_{\lambda}(y) & =\sum_{i=1}^{d} \sum_{h \in H}\left(\lambda h g_{i}, \lambda\right) \sum_{h^{\prime} \in H} \varphi\left(z^{h^{\prime} h g_{i}}\right)=|H| \sum_{i=1}^{d} \sum_{k \in H}\left(\lambda g_{i}, \lambda\right) \varphi\left(z^{k g_{i}}\right) \\
& =|H| \sum_{g \in W}(\lambda g, \lambda) \varphi\left(z^{g}\right)
\end{aligned}
$$

Now, if $\left\{g_{i}\right\}$ is a set of representatives for the right cosets of $H$, then $\left\{g_{i}^{-1}\right\}$ is a set of representatives for the left cosets of $H$. Hence for any pair $(k, i) \in H \times\{1, \ldots, d\}$ there is a unique pair $(h, j) \in H \times\{1, \ldots, d\}$ such that $k g_{i}=g_{j}^{-1} h$. Moreover, if $k g_{i}$ runs exactly once through $W$, so do the elements $g_{j}^{-1} h$. This implies, since ( , ) is $W$-invariant and since $H$ stabilizes $\lambda$,

$$
\bar{S}_{\lambda}(y)=|H| \sum_{i=1}^{d} \sum_{h \in H}\left(\lambda g_{i}, \lambda\right) \varphi\left(z^{g_{i}^{-1} h}\right)=|H| \sum_{i=1}^{d} \sum_{h \in H}\left(\lambda g_{i}, \lambda\right) \varphi\left(z^{g_{i}^{-1}}\right) .
$$

This implies the assertion.

\subsection{Kanev correspondence}

In order to define the Kanev correspondence on $Y$, let $U \subset X$ denote the complement of the branch locus of $\pi$ and fix a point $\xi_{0} \in U$. Since $H$ is the stabilizer of $\lambda$, the group $W$ acts on the set $\left\{\lambda=\lambda g_{1}, \lambda g_{2}, \ldots, \lambda g_{d}\right\}$.

The group $W$ also acts on the fibre $\psi^{-1}\left(\xi_{0}\right)$. Note that $W$ does not act on the curve $Y$, since the subgroup $H$ is not necessarily a normal subgroup of $W$. However we can define the action of $W$ on the set $\psi^{-1}\left(\xi_{0}\right)$ via the monodromy at the point $\xi_{0}$ as follows: consider the fundamental group $\pi_{1}\left(U, \xi_{0}\right)$ and let $U_{Z}=\pi^{-1}(U) \subset Z$ and $U_{Y}=\psi^{-1}(U) \subset Y$. Choose a point $z \in Z$ such that $\pi(z)=\xi_{0}$. Then $\pi_{1}\left(U_{Z}, z\right)$ is a normal subgroup of $\pi_{1}\left(U, \xi_{0}\right)$ and we have the exact sequence

$$
0 \rightarrow \pi_{1}\left(U_{Z}, z\right) \rightarrow \pi_{1}\left(U, \xi_{0}\right) \rightarrow W \rightarrow 0
$$

The monodromy of the cover $\psi: U_{Y} \rightarrow U$ at the point $\xi_{0}$ gives a homomorphism

$$
\rho: \pi_{1}\left(U, \xi_{0}\right) \rightarrow \operatorname{Aut}\left(\psi^{-1}\left(\xi_{0}\right)\right)
$$

Its kernel equals $\bigcap_{y} \pi_{1}\left(U_{Y}, y\right)$ where $y$ varies over the set $\psi^{-1}\left(\xi_{0}\right)$. We note that $\pi_{1}\left(U_{Z}, z\right) \subset \pi_{1}\left(U_{Y}, y\right)$ for any $y \in \psi^{-1}\left(\xi_{0}\right)$, hence $\pi_{1}\left(U_{Z}, z\right) \subset \operatorname{ker} \rho$. Therefore the monodromy map $\rho$ factorizes through $W$,

$$
\rho: \pi_{1}\left(U, \xi_{0}\right) \rightarrow W \rightarrow \operatorname{Aut}\left(\psi^{-1}\left(\xi_{0}\right)\right)
$$


Notice that according to our definitions the monodromy action of $\pi_{1}\left(U, \xi_{0}\right)$ on the fibre $\psi^{-1}\left(\xi_{0}\right)$ is a right action.

Choosing an element in the fibre $\psi^{-1}\left(\xi_{0}\right)$ induces a bijection

$$
\left\{\lambda g_{1}, \ldots, \lambda g_{d}\right\} \stackrel{\sim}{\rightarrow} \psi^{-1}\left(\xi_{0}\right)
$$

which is $W$-equivariant according to the definitions. In the following we identify the above sets, i.e. we label the elements of $\psi^{-1}\left(\xi_{0}\right)$ by $\lambda=\lambda g_{1}, \ldots, \lambda g_{d}$.

For every point $\xi \in U$ choose a path $\gamma_{\xi}$ in $U$ connecting $\xi$ and $\xi_{0}$. The path defines a bijection

$$
\mu: \psi^{-1}(\xi) \rightarrow \psi^{-1}\left(\xi_{0}\right)=\left\{\lambda=\lambda g_{1}, \ldots, \lambda g_{d}\right\}
$$

in the following way: For any $y \in \psi^{-1}(\xi)$ denote by $\tilde{\gamma}_{y}$ the lift of $\gamma_{\xi}$ starting at $y$. If $\lambda g_{j} \in \psi^{-1}\left(\xi_{0}\right)$ denotes the end point of $\tilde{\gamma}_{y}$, set $\mu(y)=\lambda g_{j}$. Define

$K_{U, \lambda}:=\left\{(x, y) \in \psi^{-1}(U) \times \psi^{-1}(U): \psi(x)=\psi(y),(\mu(x), \mu(y))-(\lambda, \lambda)-1>0\right\}$,

and let $\bar{K}_{\lambda}$ denote the closure of $K_{U, \lambda}$ in $Y \times Y$.

Lemma 3.2. The divisor $\bar{K}_{\lambda}$ is an integral symmetric effective correspondence on the curve $Y$, canonically associated to the triple $\left(\pi, \mathbb{S}_{\omega}, \lambda\right)$.

Proof. For the first assertion it suffices to show that $(\lambda g, \lambda)-(\lambda, \lambda)-1$ is a non-negative integer for all $g \in W \backslash H$, since (, ) is a $W$-invariant scalar product. But $(\lambda g, \lambda)-(\lambda, \lambda)-1$ is an integer, since $\lambda$ is a weight. Hence it suffices to show that $(\lambda g, \lambda)>(\lambda, \lambda)$ for every $g \in W \backslash H$. For this note that

$$
(\lambda, \lambda)-(\lambda g, \lambda)=\frac{1}{2}[(\lambda g, \lambda g)-2(\lambda g, \lambda)+(\lambda, \lambda)]=\frac{1}{2}(\lambda g-\lambda, \lambda g-\lambda),
$$

which implies the assertion, since (, ) is negative definite and $H=\operatorname{Stab}(\lambda) \subset W$.

For the last assertion we have to show that $K_{U, \lambda}$ does not depend on the choice of $\xi_{0}$ and of the path $\gamma_{\xi}$ connecting $\xi$ and $\xi_{0}$. This is a consequence of the $W$-invariance of the form $($, ) and the above mentioned fact that the monodromy map $\rho$ factorizes through the group $W$.

We call $\bar{K}_{\lambda}$ the Kanev correspondence associated to the weight $\lambda$, since it was introduced by Kanev in [K1]. Considered as a map $Y \rightarrow \operatorname{Div}(Y)$, it is given by

$$
\bar{K}_{\lambda}(y)=\sum_{j=1}^{d}\left[\left(\lambda g_{j}, \mu(y)\right)-(\lambda, \lambda)-1\right] \mu^{-1}\left(\lambda g_{j}\right)+y .
$$

Note that $y$ is added, since $y$ appears in the sum with coefficient -1 , because $\left(\lambda g_{i}, \mu(y)\right)$ $=(\lambda, \lambda)$ if $\mu(y)=\lambda g_{i}$. We need the following description of $\bar{K}_{\lambda}(y)$. According to our construction, for any $y \in \psi^{-1}(U)$, there is a unique integer $i_{y}, 1 \leq i_{y} \leq d$, such that $\mu(y)=\lambda g_{i_{y}}$. 
Proposition 3.3. If $y \in \psi^{-1}(U)$ with $\mu(y)=\lambda g_{i_{y}}$, then

$$
\bar{K}_{\lambda}(y)=\sum_{j=2}^{d}\left[\left(\lambda g_{j}, \lambda\right)-(\lambda, \lambda)-1\right] \mu^{-1}\left(\lambda g_{j} g_{i_{y}}\right) .
$$

Proof. By (7) and using the $W$-invariance of (, ), we have

$$
\begin{aligned}
\bar{K}_{\lambda}(y) & =\sum_{\lambda g_{j} \neq \mu(y)}\left[\left(\lambda g_{j}, \mu(y)\right)-(\lambda, \lambda)-1\right] \mu^{-1}\left(\lambda g_{j}\right) \\
& =\sum_{\lambda g_{j} \neq \mu(y)}\left[\left(\lambda g_{j} g_{i_{y}}^{-1}, \mu(y) g_{i_{y}}^{-1}\right)-(\lambda, \lambda)-1\right] \mu^{-1}\left(\lambda g_{j}\right) \\
& =\sum_{\lambda g_{j} \neq \lambda g_{i_{y}}}\left[\left(\lambda g_{j} g_{i_{y}}^{-1}, \lambda\right)-(\lambda, \lambda)-1\right] \mu^{-1}\left(\lambda g_{j}\right) \\
& =\sum_{j=2}^{d}\left[\left(\lambda g_{j}, \lambda\right)-(\lambda, \lambda)-1\right] \mu^{-1}\left(\lambda g_{j} g_{i_{y}}\right)
\end{aligned}
$$

\subsection{Relations between $\bar{K}_{\lambda}$ and $\bar{S}_{\lambda}$}

We want to work out how the Kanev correspondence $\bar{K}_{\lambda}$ is related to the Schur correspondence $\bar{S}_{\lambda}$ on the curve $Y$. For this we need a special choice of representatives $g_{1}=1, \ldots, g_{d}$ of the right cosets of the subgroup $H$ of $W$. We choose them so that $\left\{g_{1}^{-1}, \ldots, g_{d}^{-1}\right\}$ is also a set of representatives of the right cosets of $H$ in $W$. That there is always such a set, is a consequence of the marriage theorem of combinatorics (see [Ha, Theorem 5.1.7]).

Proposition 3.4. Let $y \in \psi^{-1}(U)$ with $\mu(y)=\lambda g_{i_{y}}$. Then

$$
\bar{S}_{\lambda}(y)=|H|^{2} \sum_{j=1}^{d}\left(\lambda g_{j}, \lambda\right) \mu^{-1}\left(\lambda g_{j} g_{i_{y}}\right) .
$$

Proof. According to Proposition 3.1 $\bar{S}_{\lambda}(y)=|H|^{2} \sum_{j=1}^{d}\left(\lambda g_{j}, \lambda\right) \varphi\left(z^{g_{j}^{-1}}\right)$, where $z$ is a point in the fiber $\varphi^{-1}(y) \subset Z$.

Suppose first that $\mu(y)=\lambda$, i.e. $i_{y}=1$. As $W$ acts by right multiplication on $\left\{\lambda g_{1}, \ldots, \lambda g_{d}\right\}$, we have $\mu(\varphi(z)) g=\mu\left(\varphi\left(z^{g}\right)\right)$, and hence

$$
\varphi\left(z^{g_{j}^{-1}}\right)=\mu^{-1}\left(\mu(\varphi(z)) g_{j}^{-1}\right)=\mu^{-1}\left(\lambda g_{j}^{-1}\right)
$$

for all $j$. Therefore, by the $W$-invariance of (, ),

$$
\begin{aligned}
\bar{S}_{\lambda}(y) & =|H|^{2} \sum_{j=1}^{d}\left(\lambda g_{j}, \lambda\right) \mu^{-1}\left(\lambda g_{j}^{-1}\right)=|H|^{2} \sum_{j=1}^{d}\left(\lambda, \lambda g_{j}^{-1}\right) \mu^{-1}\left(\lambda g_{j}^{-1}\right) \\
& =|H|^{2} \sum_{j=1}^{d}\left(\lambda g_{j}, \lambda\right) \mu^{-1}\left(\lambda g_{j}\right),
\end{aligned}
$$


where for the last equality we have used the fact that together with $\left\{g_{1}, \ldots, g_{d}\right\}$ also $\left\{g_{1}^{-1}, \ldots, g_{d}^{-1}\right\}$ is a set of representatives of the right cosets of $H$.

Finally, if $\mu(y) \neq \lambda$, we have $\mu(y) g_{i_{y}}^{-1}=\lambda$. Hence we can apply the above equality to the bijection $\widetilde{\mu}=g_{i_{y}}^{-1} \cdot \mu: \psi^{-1}(\psi(y)) \rightarrow\left\{\lambda g_{1}, \ldots, \lambda g_{d}\right\}$, which gives

$$
\bar{S}_{\lambda}(y)=|H|^{2} \sum_{j=1}^{d}\left(\lambda g_{j}, \lambda\right) \tilde{\mu}^{-1}\left(\lambda g_{j}\right)=|H|^{2} \sum_{j=1}^{d}\left(\lambda g_{j}, \lambda\right) \mu^{-1}\left(\lambda g_{j} g_{i_{y}}\right) .
$$

With the above notation and identifications we can state the main result of this section.

Theorem 3.5. The Kanev and Schur correspondences $\bar{K}_{\lambda}$ and $\bar{S}_{\lambda}$ on the curve $Y=Z / H$ associated to $\lambda$ are related as follows:

$$
\bar{S}_{\lambda}=|H|^{2}\left(\bar{K}_{\lambda}-\bar{\Delta}+((\lambda, \lambda)+1) \bar{T}\right),
$$

where $\bar{\Delta}$ denotes the diagonal in $Y \times Y$ and $\bar{T}=\psi^{*} \psi_{*}$ is the trace correspondence of the morphism $\psi$.

Proof. It suffices to show that $\left[\bar{S}_{\lambda}+|H|^{2}\left(\bar{\Delta}-\bar{K}_{\lambda}\right)\right](y)=[(\lambda, \lambda)+1]|H|^{2} \bar{T}(y)$ for all $y \in \psi^{-1}(U)$. But applying Propositions 3.3 and 3.4 we have

$$
\begin{aligned}
{\left[\bar{S}_{\lambda}+|H|^{2}\left(\bar{\Delta}-\bar{K}_{\lambda}\right)\right](y)=|H|^{2}\left[\sum_{j=1}^{d}\left(\lambda g_{j}, \lambda\right) \mu^{-1}\left(\lambda g_{j} g_{i_{y}}\right)+y\right.} \\
\left.-\sum_{j=2}^{d}\left[\left(\lambda g_{j}, \lambda\right)-(\lambda, \lambda)-1\right] \mu^{-1}\left(\lambda g_{j} g_{i_{y}}\right)\right] \\
=|H|^{2}((\lambda, \lambda)+1) \sum_{j=1}^{d} \mu^{-1}\left(\lambda g_{j} g_{i_{y}}\right) .
\end{aligned}
$$

But right multiplication with $g_{i_{y}}$ permutes only the elements $\lambda g_{1}, \ldots, \lambda g_{d}$, which implies that $\sum_{j=1}^{d} \mu^{-1}\left(\lambda g_{j} g_{i_{y}}\right)=\psi^{-1} \psi(y)=\bar{T}(y)$. This completes the proof.

Corollary 3.6. $\operatorname{deg} \bar{K}_{\lambda}=1-d((\lambda, \lambda)+1)$.

Proof. Note first that deg $S_{\lambda}=\sum_{g \in W}(\lambda g, \lambda)=\left(\sum_{g \in W} \lambda g, \lambda\right)=0$, since $\sum_{g \in W} \lambda g$ is $W$-invariant and thus equal to 0 in $V$. This implies $\operatorname{deg} \bar{S}_{\lambda}=0$, and hence Theorem 3.5 gives the assertion, since $\operatorname{deg} \bar{\Delta}=1$ and $\operatorname{deg} \bar{T}=d$.

Corollary 3.7. We have the relations

(1) $\bar{T} \bar{S}_{\lambda}=\bar{S}_{\lambda} \bar{T}=0$,

(2) $\bar{K}_{\lambda} \bar{T}=\bar{T} \bar{K}_{\lambda}=\operatorname{deg} \bar{K}_{\lambda} \cdot \bar{T}$. 
Proof. To obtain (1) we consider $T=\pi^{*} \pi_{*}$, the trace correspondence of $\pi$, and note that for all $z \in Z$,

$$
T S_{\lambda}(z)=\sum_{g \in W}(\lambda g, \lambda) T\left(z^{g}\right)=\sum_{g \in W}(\lambda g, \lambda) \cdot T(z)=\operatorname{deg} S_{\lambda} \cdot T(z)=0
$$

and similarly $S_{\lambda} T=0$. Now $\bar{T}=(1 /|H|) \varphi_{*} T$ implies the assertion.

To obtain (2), using $\bar{\Delta} \bar{T}=\bar{T}, \bar{T}^{2}=d \bar{T}$ and Theorem 3.5 we compute

$$
\bar{K}_{\lambda} \bar{T}=\frac{1}{|H|^{2}} \bar{S}_{\lambda} \bar{T}+\bar{\Delta} \bar{T}-((\lambda, \lambda)+1) \bar{T}^{2}=\bar{T}-d((\lambda, \lambda)+1) \bar{T}=\operatorname{deg} \bar{K}_{\lambda} \cdot \bar{T},
$$

where the last equality follows from Corollary 3.6 Similarly Theorem 3.5 gives $\bar{K}_{\lambda} \bar{T}=$ $\bar{T} \bar{K}_{\lambda}$.

For the next theorem we need a lemma.

Lemma 3.8. $\bar{S}_{\lambda}^{2}=\bar{e} \cdot \bar{S}_{\lambda}$, with $\bar{e}=|H| \cdot|W| \cdot(\lambda, \lambda) / \operatorname{dim} V_{\omega} \in \mathbb{Q}$.

Proof. Schur's orthogonality relations imply that $p_{\lambda}=(1 / e) \cdot S_{\lambda}$ is an idempotent of the rational group ring $\mathbb{Q}[W]$ or equivalently that $S_{\lambda}^{2}=e \cdot S_{\lambda}$ with $e=|W| \cdot(\lambda, \lambda) / \operatorname{dim} V_{\omega}$ (see [LR, Proposition 2.3]). Note that $e<0$, since (, ) is negative definite.

According to [Fu, Proposition 16.1.2(a)], $(\varphi \times \varphi)_{*} S_{\lambda} \cdot(\varphi \times \varphi)_{*} S_{\lambda}=(\varphi \times \varphi)_{*}\left(S_{\lambda} \cdot S_{\lambda}\right)$, which implies

$$
\bar{S}_{\lambda}^{2}=(\varphi \times \varphi)_{*}\left(S_{\lambda}^{2}\right)=(\varphi \times \varphi)_{*}\left(e \cdot S_{\lambda}\right)=(\varphi \times \varphi)_{*}(e \Delta) \cdot(\varphi \times \varphi)_{*}\left(S_{\lambda}\right)=|H| e \bar{S}_{\lambda} .
$$

Theorem 3.9. The Kanev correspondence $\bar{K}_{\lambda}$ satisfies the cubic equation

$$
\left(\bar{K}_{\lambda}-\bar{\Delta}\right)\left(\bar{K}_{\lambda}+\left(q_{\lambda}-1\right) \bar{\Delta}\right)\left(\bar{K}_{\lambda}-\operatorname{deg} \bar{K}_{\lambda} \bar{\Delta}\right)=0
$$

with $q_{\lambda}=-d \cdot(\lambda, \lambda) / \operatorname{dim} V_{\omega} \in \mathbb{N}$.

Proof. Theorem 3.5 and Lemma 3.8 give

$$
|H|^{4}\left(\bar{K}_{\lambda}-\bar{\Delta}+a \bar{T}\right)^{2}=\bar{e}|H|^{2}\left(\bar{K}_{\lambda}-\bar{\Delta}+a \bar{T}\right)
$$

with $a=(\lambda, \lambda)+1$. Applying Corollary 3.7 and $\bar{T}^{2}=d \bar{T}$ as well as $\bar{\Delta} \bar{T}=\bar{T}$ shows that there is a rational number $c$ such that

$$
\left(\bar{K}_{\lambda}-\bar{\Delta}\right)^{2}-\frac{\bar{e}}{|H|^{2}}\left(\bar{K}_{\lambda}-\bar{\Delta}\right)+c \bar{T}=0 .
$$

Multiplying this equation by $\bar{K}_{\lambda}-\operatorname{deg} \bar{K}_{\lambda} \bar{\Delta}$ and using Corollary 3.7 again, we get

$$
\left(\bar{K}_{\lambda}-\bar{\Delta}\right)\left(\bar{K}_{\lambda}-\bar{\Delta}-\frac{\bar{e}}{|H|^{2}} \bar{\Delta}\right)\left(\bar{K}_{\lambda}-\operatorname{deg} \bar{K}_{\lambda} \bar{\Delta}\right)=0 .
$$

This completes the proof of the theorem. 


\subsection{The Prym variety $P_{\lambda}$}

We consider the rational correspondence $\bar{s}_{\lambda}=\left(1 /|H|^{2}\right) \bar{S}_{\lambda}$. Then $\bar{s}_{\lambda}$ satisfies the relation $\bar{s}_{\lambda}^{2}=-q_{\lambda} \bar{s}_{\lambda}$ and by Theorem 3.5 we have

$$
\bar{s}_{\lambda}=\bar{K}_{\lambda}-\bar{\Delta}+((\lambda, \lambda)+1) \bar{T} .
$$

Hence we see that $\bar{s}_{\lambda}$ is an integral correspondence if and only if $(\lambda, \lambda)$ is an integer. This happens e.g. for $E_{8}$, but not for $E_{6}$ or $E_{7}$-see the tables in Section 4.

The correspondence $\bar{s}_{\lambda}$ induces a rational endomorphism also denoted by $\bar{s}_{\lambda} \in$ $\operatorname{End}_{\mathbb{Q}}(\operatorname{Jac}(Y))$. We introduce the following abelian subvariety of $\operatorname{Jac}(Y)$, which we will also call the Prym variety:

$$
P_{\lambda}:=\operatorname{im}\left(m \bar{s}_{\lambda}\right) \subset \operatorname{Jac}(Y),
$$

where $m$ is some integer such that $m \bar{s}_{\lambda} \in \operatorname{End}(\operatorname{Jac}(Y))$. It is clear that $P_{\lambda}$ does not depend on the integer $m$.

Since $\bar{K}_{\lambda}-\bar{\Delta}$ is a correspondence on $Y$ it induces an endomorphism $v_{\lambda} \in$ $\operatorname{End}(\operatorname{Jac}(Y))$. We denote by

$$
S=\operatorname{Prym}(Y / X)
$$

the Prym variety of the covering $\psi: Y \rightarrow X$ (see Remark 2.1(ii)). Since by Corollary 3.7 $v_{\lambda} t=t v_{\lambda}$, we see that $v_{\lambda}(S) \subset S$. Hence $v_{\lambda}$ restricts to a symmetric endomorphism

$$
u_{\lambda} \in \operatorname{End}(S) \quad \text { with } \quad u_{\lambda}^{2}=q_{\lambda} u_{\lambda} .
$$

Proposition 3.10. We have an equality of abelian subvarieties of $S$,

$$
P_{\lambda}=\operatorname{im} u_{\lambda} \subset S .
$$

Proof. By (8) the endomorphisms $\bar{s}_{\lambda}$ and $u_{\lambda}$ coincide on $S$ up to a non-zero integer multiple. Moreover, the restriction of $\bar{s}_{\lambda}$ to the subvariety $\psi^{*} \operatorname{Jac}(X)$ is zero because $\bar{s}_{\lambda} t=0$ (Corollary 3.7). The equality now follows since $\operatorname{Jac}(Y)$ and $\operatorname{Jac}(X) \times S$ are isogenous.

Remark 3.11. Note that the abelian subvariety $P_{\lambda} \subset S$ only depends on $\lambda \in V_{\omega}$ and not on the lattice $\mathbb{S}_{\omega} \subset V_{\omega}$. Moreover, we recall from [Me, Proposition 4.3(1)] that $P_{\lambda}$ and $P_{\lambda^{\prime}}$ are isogenous for any $\lambda, \lambda^{\prime} \in V_{\omega}$.

Remark 3.12. If $\psi: Y \rightarrow X$ is étale, then the Kanev correspondence $\bar{K}_{\lambda}$ is fixed-pointfree. On the other hand, we notice that the induced endomorphism $v_{\lambda}$ does not satisfy a quadratic equation - only its restriction $u_{\lambda}$ to $S$, hence we are not in a situation where

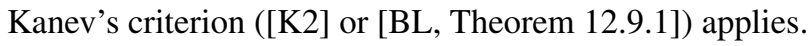

\section{Examples}

As our main examples let us work out the invariants of the last section in the case of a Weyl group $W$ of ADE-type. Let $\pi: Z \rightarrow X$ be a Galois covering of smooth projective 
curves with Galois group $W$. As weights $\lambda$ we choose the minuscule weights $\varpi_{i}$ (in the notation of $[\mathrm{Bo}]$ ) except in the case of type $E_{8}$, where we consider the quasi-minuscule weight $\lambda=\varpi_{8}$. As above, let $V_{\lambda}$ denote the corresponding absolutely irreducible $\mathbb{Q}$ representation, $H$ the stabilizer subgroup of $\lambda$ in $W$, and $Y=Z / H$. If ( , ) denotes the uniquely determined $W$-invariant negative definite symmetric form on $V_{\lambda}$ as defined in Section 3.2, the following tables give the values for the numbers $(\lambda, \lambda), d=\operatorname{deg}(Y / X)$, $\operatorname{dim} V_{\lambda}, q_{\lambda}$ and the degree of the Kanev correspondence $\bar{K}_{\lambda}$. For the definition of the Dynkin index $d_{\lambda}$ see [LS] and also Proposition 7.4. The case of a Weyl group of type $E$ is closely related to a del Pezzo surface (see [K1]). The last line of the first table gives the degree of the corresponding surface.

\begin{tabular}{c|ccccc}
\hline Weyl group of type & $E_{4}=A_{4}$ & $E_{5}=D_{5}$ & $E_{6}$ & $E_{7}$ & $E_{8}$ \\
\hline weight $\lambda$ & $\varpi_{2}$ & $\varpi_{4}, \varpi_{5}$ & $\varpi_{1}, \varpi_{6}$ & $\varpi_{7}$ & $\varpi_{8}$ \\
$(\lambda, \lambda)$ & $-\frac{6}{5}$ & $-\frac{5}{4}$ & $-\frac{4}{3}$ & $-\frac{3}{2}$ & -2 \\
$d=\operatorname{deg}(Y / X)$ & 10 & 16 & 27 & 56 & 240 \\
$\operatorname{dim} V_{\lambda}$ & 10 & 16 & 27 & 56 & 248 \\
$q_{\lambda}=d_{\lambda}$ & 3 & 4 & 6 & 12 & 60 \\
$\operatorname{deg} \bar{K}_{\lambda}$ & 3 & 5 & 10 & 29 & 241 \\
del Pezzo of degree & 5 & 4 & 3 & 2 & 1 \\
\hline
\end{tabular}

\begin{tabular}{c|cc}
\hline Weyl group of type & $A_{n}$ & $D_{n}$ \\
\hline weight $\lambda$ & $\varpi_{i}, 1 \leq i \leq n$ & $\varpi_{n-1}, \varpi_{n}$ \\
$(\lambda, \lambda)$ & $-\frac{i(n+1-i)}{n+1}$ & $-\frac{n}{4}$ \\
$d=\operatorname{deg}(Y / X)$ & $\left(\begin{array}{c}n+1 \\
i\end{array}\right)$ & $2^{n-1}$ \\
$\operatorname{dim} V_{\lambda}$ & $\left(\begin{array}{c}n+1 \\
i\end{array}\right)$ & $2^{n-1}$ \\
$q_{\lambda}=d_{\lambda}$ & $\left(\begin{array}{c}n-1 \\
i-1\end{array}\right)$ & $2^{n-3}$ \\
$\operatorname{deg} \bar{K}_{\lambda}$ & $\left(\begin{array}{c}n-1 \\
i-1\end{array}\right) n-\left(\begin{array}{c}n+1 \\
i\end{array}\right)+1$ & $2^{n-3}(n-4)+1$ \\
\hline
\end{tabular}

Proof. The symmetric form ( , ) is given by the negative of the Cartan matrix. This gives the value of $(\lambda, \lambda)$ using the explicit form of $\lambda$ as outlined in [Bo]. The length of the orbit of $\lambda$ under the action of $W$ gives the degree of $Y / X$. The dimension of $V_{\lambda}$ follows from the fact that $\lambda$ is a minuscule weight, respectively quasi-minuscule weight in the case of $E_{8}$. The values of $q_{\lambda}$ and $\operatorname{deg} \bar{K}_{\lambda}$ are computed by Theorem 3.9 and Corollary 3.6. For the degree of the del Pezzo surface see [K1]. For the Dynkin indices see [LS, Proposition 2.6].

Remark 4.1. We note that in the examples of the tables $q_{\lambda}=d_{\lambda}$. This equality of the two integers is a coincidence, as e.g. for $\left(G_{2}, \varpi_{1}\right): d_{\lambda}=2, q_{\lambda}=6$, or for $\left(F_{4}, \varpi_{4}\right): d_{\lambda}=6$, $q_{\lambda}=12$ (see [LS, Proposition 2.6] and [K1, p. 176]). 


\section{The Donagi-Prym variety}

\subsection{Definitions and properties}

Following [Don1] we introduce the compact commutative algebraic group

$$
\operatorname{Prym}\left(\pi, \mathbb{S}_{\omega}\right):=\operatorname{Hom}_{W}\left(\mathbb{S}_{\omega}, \operatorname{Pic}(Z)\right),
$$

which we call the Donagi-Prym variety associated to the pair $\left(\pi, \mathbb{S}_{\omega}\right)$, where $\pi: Z \rightarrow X$ is a Galois covering with group $W$ and $\mathbb{S}_{\omega}$ is a right $\mathbb{Z}[W]$-module. The elements of $\operatorname{Prym}\left(\pi, \mathbb{S}_{\omega}\right)$ are $W$-equivariant homomorphisms $\phi: \mathbb{S}_{\omega} \rightarrow \operatorname{Pic}(Z)$; for an alternative description see Lemma 6.1. Its connected component $\operatorname{Prym}\left(\pi, \mathbb{S}_{\omega}\right)_{0}$ containing 0 is an abelian variety. For any element $\lambda \in \mathbb{S}_{\omega}$ we consider the corresponding evaluation map

$$
\operatorname{ev}_{\lambda}: \operatorname{Prym}\left(\pi, \mathbb{S}_{\omega}\right) \rightarrow \operatorname{Pic}(Z), \quad \phi \mapsto \phi(\lambda)
$$

Proposition 5.1. We have $\operatorname{Hom}_{W}\left(\mathbb{S}_{\omega}, \operatorname{Pic}(Z)\right)=\operatorname{Hom}_{W}\left(\mathbb{S}_{\omega}, \operatorname{Jac}(Z)\right)$. In particular, all connected components of the image $\operatorname{ev}_{\lambda}\left(\operatorname{Prym}\left(\pi, \mathbb{S}_{\omega}\right)\right)$ are contained in $\operatorname{Jac}(Z)$.

Proof. Consider a homomorphism $\phi \in \operatorname{Hom}_{W}\left(\mathbb{S}_{\omega}\right.$, Pic $\left.(Z)\right)$. The composite map deg $\circ \phi$ is a $W$-invariant homomorphism from $\mathbb{S}_{\omega}$ to $\mathbb{Z}$. Since the dual lattice $\operatorname{Hom}_{\mathbb{Z}}\left(\mathbb{S}_{\omega}, \mathbb{Z}\right)$ is an irreducible $W$-module, we conclude that deg $\circ \phi=0$.

We define

$$
\Gamma_{\lambda}=\mathbb{S}_{\omega} /(\lambda \cdot \mathbb{Z}[W]) .
$$

This is a finite abelian group since $V_{\omega}=\mathbb{S}_{\omega} \otimes_{\mathbb{Z}} \mathbb{Q}$ is an irreducible $W$-module.

Proposition 5.2. $\operatorname{ker}_{\lambda}=\operatorname{Hom}_{W}\left(\Gamma_{\lambda}, \operatorname{Jac}(Z)\right)$.

Proof. Let $U$ denote the image of the natural map $\mathbb{Z}[W] \stackrel{a}{\rightarrow} U \stackrel{i}{\rightarrow} \mathbb{S}_{\omega}$ defined by $a(g)=$ $\lambda g$ for $g \in W$. Then we have the exact sequence of right $\mathbb{Z}[W]$-modules

$$
0 \rightarrow U \stackrel{i}{\rightarrow} \mathbb{S}_{\omega} \rightarrow \Gamma_{\lambda} \rightarrow 0
$$

Now we apply the left-exact contravariant functor $\operatorname{Hom}_{W}(\cdot, \operatorname{Jac}(Z))$ to obtain the exact sequence

$$
0 \rightarrow \operatorname{Hom}_{W}\left(\Gamma_{\lambda}, \operatorname{Jac}(Z)\right) \rightarrow \operatorname{Hom}_{W}\left(\mathbb{S}_{\omega}, \operatorname{Jac}(Z)\right) \stackrel{\check{i}}{\longrightarrow} \operatorname{Hom}_{W}(U, \operatorname{Jac}(Z)) .
$$

We then apply the same functor to the surjective $\mathbb{Z}[W]$-morphism $a: \mathbb{Z}[W] \rightarrow U$ and we obtain an injective morphism

$$
\check{a}: \operatorname{Hom}_{W}(U, \operatorname{Jac}(Z)) \rightarrow \operatorname{Hom}_{W}(\mathbb{Z}[W], \operatorname{Jac}(Z))=\operatorname{Jac}(Z) .
$$

Note that $\check{a} \circ \check{i}=\operatorname{ev}_{\lambda}$, and $\operatorname{ker} \operatorname{ev}_{\lambda}=\operatorname{ker} \check{i}$ because $\check{a}$ is injective. 


\subsection{The image of the evaluation map $\mathrm{ev}_{\lambda}$}

It is clear that $\operatorname{ev}_{\lambda}\left(\operatorname{Prym}\left(\pi, \mathbb{S}_{\omega}\right)\right) \subset \operatorname{Jac}^{H}(Z)$, where $H=\operatorname{Stab}(\lambda)$ and $\operatorname{Jac}^{H}(Z)$ denotes the subvariety of $\operatorname{Jac}(Z)$ parametrizing $H$-invariant line bundles over $Z$. We recall the exact sequence of abelian groups ([Dol, Proposition 2.2])

$$
0 \rightarrow H^{*} \rightarrow \operatorname{Pic}(H ; Z) \stackrel{\iota}{\longrightarrow} \operatorname{Pic}^{H}(Z) \rightarrow H^{2}\left(H, \mathbb{C}^{*}\right) \rightarrow 0,
$$

where $\operatorname{Pic}(H ; Z)$ denotes the group of $H$-linearized line bundles over $Z$ (see [Dol, Section 1]) and $H^{*}=\operatorname{Hom}\left(H, \mathbb{C}^{*}\right)$ denotes the group of characters of $H$. The map $\iota$ is the map which forgets the $H$-linearization.

Proposition 5.3. We have the exact sequence

$$
0 \rightarrow H^{*} \rightarrow \operatorname{Jac}(H ; Z) \stackrel{\iota}{\longrightarrow} \operatorname{Jac}^{H}(Z) \rightarrow H^{2}\left(H, \mathbb{C}^{*}\right) \rightarrow 0 .
$$

Proof. We denote by $d$ the degree of the cover $\psi: Y \rightarrow X$. Consider the following diagram, in which the first line is obtained as the kernel of the degree morphism:

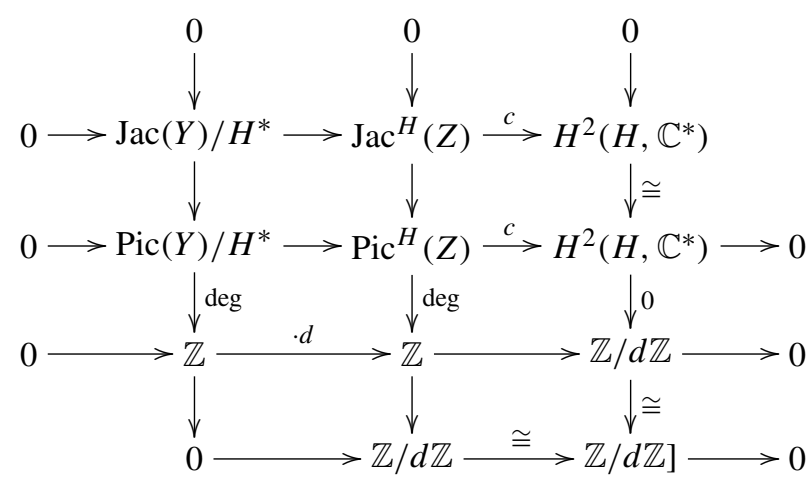

The snake lemma now implies that the natural homomorphism $c: \operatorname{Jac}^{H}(Z) \rightarrow H^{2}\left(H, \mathbb{C}^{*}\right)$ is surjective.

In Section 6.5 we will show that for any $\lambda \in \mathbb{S}_{\omega}$ the evaluation map $\operatorname{ev}_{\lambda} \operatorname{lifts}$ to $\operatorname{Jac}(H ; Z)$. Finally, we also recall the exact sequence

$$
0 \rightarrow \operatorname{Jac}(Y) \rightarrow \operatorname{Jac}(H ; Z) \rightarrow \bigoplus_{x \in \operatorname{Br}(\varphi)} \mathbb{Z} / e_{x} \mathbb{Z} \rightarrow 0,
$$

where $x$ varies over the branch divisor $\operatorname{Br}(\varphi)$ of the Galois covering $\varphi: Z \rightarrow Y=Z / H$ and $e_{x}$ denotes the ramification index of $x$.

\subsection{The group $\Gamma_{\lambda}$}

We now compute the finite group $\Gamma_{\lambda}$ in some special cases, which will be worked out in detail in Section 8. Given a simple Lie algebra $\mathfrak{g}$ we take for $\mathbb{S}_{\omega}$ the weight lattice of $\mathfrak{g}$ and denote by $\Lambda_{R} \subset \mathbb{S}_{\omega}$ the root lattice. We use the notation of [Bo]. In particular, $\alpha_{i}$ denote the simple roots and $\varpi_{i}$ denote the fundamental weights of $\mathfrak{g}$. 
Lemma 5.4. In the following cases the group $\Gamma_{\lambda}$ is trivial:

(1) $W=W\left(E_{8}\right), \lambda=\varpi_{8}$.

(2) $W=W\left(E_{7}\right), \lambda=\varpi_{7}$.

(3) $W=W\left(E_{6}\right), \lambda=\varpi_{1}$ or $\lambda=\varpi_{6}$.

(4) $W=W\left(D_{n}\right)$ with $n$ odd, $\lambda=\varpi_{n-1}$ or $\lambda=\varpi_{n}$.

(5) $W=W\left(A_{n}\right), \lambda=\varpi_{i}$ with $i$ coprime to $n+1$.

Proof. In each case we have to show that $\lambda \cdot \mathbb{Z}[W]=\mathbb{S}_{\omega}$. Let $\alpha \in \Lambda_{R}$ be a root and let $s_{\alpha}$ be its associated reflection. We have

$$
s_{\alpha}(\varpi)=\varpi-(\varpi \mid \alpha) \alpha \quad \forall \varpi \in \mathbb{S}_{\omega} .
$$

Note that for Lie algebras of type $\mathrm{ADE}$ all roots $\alpha$ have the same length $(\alpha \mid \alpha)=2$. Here (.|.) denotes the Cartan-Killing form on $\Lambda_{R}$.

(1) Clearly the elements $\varpi_{8}$ and $s_{\alpha_{8}}\left(\varpi_{8}\right)$ are in the lattice $\lambda \cdot \mathbb{Z}[W]$. Moreover, $s_{\alpha_{8}}\left(\varpi_{8}\right)=\varpi_{8}-\alpha_{8}$, hence $\alpha_{8} \in \lambda \cdot \mathbb{Z}[W]$. The Weyl group $W$ acts transitively on the roots, which implies that $\Lambda_{R} \subset \lambda \cdot \mathbb{Z}[W]$. For $\mathfrak{g}$ of type $E_{8}$, we have $\Lambda_{R}=\mathbb{S}_{\omega}$ and we are done.

(2) As before $\varpi_{7}, s_{\alpha_{7}}\left(\varpi_{7}\right) \in \lambda \cdot \mathbb{Z}[W]$, hence $\alpha_{7} \in \lambda \cdot \mathbb{Z}[W]$. Since $W$ acts transitively on the roots, we have $\Lambda_{R} \subset \lambda \cdot \mathbb{Z}[W]$. Here $\varpi_{7} \in \lambda \cdot \mathbb{Z}[W]$, but $\varpi_{7} \notin \Lambda_{R}$. Since $\left[\mathbb{S}_{\omega}: \Lambda_{R}\right]=2$, this implies the assertion.

(3) The computations are similar to the previous case and use $\left(\alpha_{1} \mid \varpi_{1}\right)=\left(\alpha_{6} \mid \varpi_{6}\right)=1$ and $\left[\mathbb{S}_{\omega}: \Lambda_{R}\right]=3$.

(4) Since $W$ acts transitively on the roots, we see as before that $\Lambda_{R} \subset \lambda \cdot \mathbb{Z}[W]$. Since $n$ is odd, we find that $\mathbb{S}_{\omega} / \Lambda_{R} \cong \mathbb{Z} / 4 \mathbb{Z}$ is generated by the class of $\varpi_{n}$ or $\varpi_{n-1}$.

(5) As before, $\Lambda_{R} \subset \lambda \cdot \mathbb{Z}[W]$. The class of the fundamental weight $\varpi_{i}$ in the quotient $\mathbb{S}_{\omega} / \Lambda_{R} \cong \mathbb{Z} /(n+1) \mathbb{Z}$ equals the class of $i \in \mathbb{Z} /(n+1) \mathbb{Z}$. The assertion then follows.

\section{Mumford groups}

\subsection{Twisted Mumford groups}

Let $T$ be a torus and suppose we are given a left action $\sigma: W \rightarrow \operatorname{Aut}(T)$ of the group $W$ on $T$. For any $g \in W$ we also denote the automorphism of $T$ by $g$. This left action $\sigma$ induces a right action of $W$ on the group of characters $\operatorname{Hom}\left(T, \mathbb{C}^{*}\right)$. We suppose that the representation of $W$ on $\operatorname{Hom}\left(T, \mathbb{C}^{*}\right) \otimes_{\mathbb{Z}} \mathbb{Q}$ is irreducible, hence $\operatorname{Hom}\left(T, \mathbb{C}^{*}\right)$ is of the form $\mathbb{S}_{\omega}$ for some $\omega \in \widehat{W}$. Conversely, given a lattice $\mathbb{S}_{\omega}$ there always exists a torus $T$ with a left $W$-action such that $\mathbb{S}_{\omega}=\operatorname{Hom}\left(T, \mathbb{C}^{*}\right)$ as $W$-modules. We recall that $W$ acts from the right on the curve $Z$.

Let $E_{T}$ be a principal $T$-bundle over $Z$. The group $W$ then acts from the left on the set of principal $T$-bundles in two different ways: $g \in W$ sends $E_{T}$ to

(1) $g^{*} E_{T}$, the pull-back of $E_{T}$ under the automorphism $g$ of $Z$;

(2) $E_{T} \times{ }_{g}^{T} T$, the $T$-bundle obtained from $E_{T}$ by extension of structure group from $T$ to $T$, where $t \in T$ acts on $T$ by left multiplication with $g(t) \in T$. 
Note that both actions commute. In this paper we will denote the combined left $W$-action on the set of principal $T$-bundles by

$$
\text { g. } E_{T}=g^{*} E_{T} \times{ }_{g}^{T} T \quad \text { for } g \in W .
$$

Since the action of $W$ on $Z$ is a right action, we have $\left(g_{1} g_{2}\right)^{*} E_{T}=g_{1}^{*} g_{2}^{*} E_{T}$. This implies that $\left(g, E_{T}\right) \mapsto g . E_{T}$ is a left action.

We say that a $T$-bundle $E_{T}$ is $W$-invariant if $E_{T} \simeq g . E_{T}$ for all $g \in W$. Here $\simeq$ denotes a $T$-equivariant isomorphism. Let $\underline{T}$ denote the sheaf of abelian groups on $Z$ defined by $\underline{T}(U)=\operatorname{Mor}(U, T)$ for any open subset $U \subset Z$. The cohomology group $H^{1}(Z, \underline{T})$ parametrizes the isomorphism classes of $T$-bundles over $Z$. We denote by $H^{1}(Z, T)^{W}$ the $W$-invariant subset, where $W$ acts both on the curve $Z$ and on the torus $T$.

Lemma 6.1. There are canonical bijections

$$
H^{1}(Z, \underline{T})^{W}=\left\{W \text {-invariant } T \text {-bundles } E_{T} \text { on } Z\right\}=\operatorname{Prym}\left(\pi, \mathbb{S}_{\omega}\right) .
$$

Proof. The first equality follows from the definition of $H^{1}(Z, \underline{T})^{W}$. For the second equality note that the map

$$
\begin{aligned}
& \Phi:\left\{T \text {-bundles } E_{T} \text { on } Z\right\} \rightarrow \operatorname{Hom}\left(\mathbb{S}_{\omega}, \operatorname{Pic}(Z)\right), \\
& \Phi\left(E_{T}\right)=\left(\phi: \mathbb{S}_{\omega} \rightarrow \operatorname{Pic}(Z), \phi(\lambda)=E_{T} \times{ }_{\lambda}^{T} \mathbb{C}^{*}\right),
\end{aligned}
$$

is bijective. Its inverse maps $\phi \in \operatorname{Hom}\left(\mathbb{S}_{\omega}, \operatorname{Pic}(Z)\right)$ to $E_{T}=L_{1} \oplus \cdots \oplus L_{n}$, where $L_{i}=\phi\left(\lambda_{i}\right), \lambda_{1}, \ldots, \lambda_{n}$ being a $\mathbb{Z}$-basis of $\mathbb{S}_{\omega}$. One can easily show that the inverse map does not depend on the choice of the $\mathbb{Z}$-basis.

We have to show that $\phi=\Phi\left(E_{T}\right)$ is $W$-equivariant if and only if $E_{T} \simeq g . E_{T}$ for all $g \in W$. As $W$ acts from the right on $\mathbb{S}_{\omega}$ and $Z$, the map $\phi$ is $W$-equivariant if and only if

$$
\phi(\lambda g)=\left(g^{-1}\right)^{*} \phi(\lambda)
$$

for all $\lambda \in \mathbb{S}_{\omega}$ and $g \in W$. But

$$
\phi(\lambda g)=E_{T} \times{ }_{\lambda g}^{T} \mathbb{C}^{*}=\left(E_{T} \times{ }_{g}^{T} T\right) \times_{\lambda}^{T} \mathbb{C}^{*}
$$

and

$$
\left(g^{-1}\right)^{*} \phi(\lambda)=\left(g^{-1}\right)^{*}\left(E_{T} \times{ }_{\lambda}^{T} \mathbb{C}^{*}\right) .
$$

Hence 111 is valid for all $\lambda \in \mathbb{S}_{\omega}$ if and only if $\left(g^{-1}\right)^{*} E_{T} \simeq E_{T} \times{ }_{g}^{T} T$, which is the case if and only if $E_{T} \simeq g^{*} E_{T} \times{ }_{g}^{T} T=g . E_{T}$.

Definition 6.2. Given a $W$-invariant $T$-bundle $E_{T}$ over $Z$ we define the $\sigma$-twisted Mumford group of $E_{T}$ by

$$
\mathcal{G}^{\sigma}\left(E_{T}\right):=\left\{(\gamma, g) \mid g \in W, \gamma: E_{T} \rightarrow g . E_{T}\right\},
$$

where $\gamma$ is a T-equivariant isomorphism. The composition law in $\mathcal{G}^{\sigma}\left(E_{T}\right)$ is given by

$$
(\gamma, g) .\left(\mu, g^{\prime}\right)=\left((g . \mu) \circ \gamma, g g^{\prime}\right)
$$

where $g . \mu: g . E_{T} \rightarrow g \cdot\left(g^{\prime} \cdot E_{T}\right)=\left(g g^{\prime}\right) \cdot E_{T}$ denotes the isomorphism obtained by applying $g$. to $\mu$. 
Since the automorphism group $\operatorname{Aut}_{T}\left(E_{T}\right)$ equals $T$, we see that the group $\mathcal{G}^{\sigma}\left(E_{T}\right)$ fits into the exact sequence

$$
1 \rightarrow T \rightarrow \mathcal{G}^{\sigma}\left(E_{T}\right) \rightarrow W \rightarrow 1,
$$

where the second map forgets the isomorphism $\gamma$. We note that the induced action by conjugation of $W$ on the normal subgroup $T$ coincides with $\sigma$. To simplify notation we denote the $\sigma$-twisted Mumford group $\mathcal{G}^{\sigma}\left(E_{T}\right)$ by $N$.

Proposition 6.3. Let $E_{T}$ be a $W$-invariant $T$-bundle over $Z$ with projection $p: E_{T} \rightarrow$ $Z$. Then the natural $T$-action on $E_{T}$ extends canonically to an $N$-action on the variety $E_{T}$ preserving the fibres of $\pi \circ p: E_{T} \rightarrow X$, i.e. there exists a morphism over $X$ defining a group action

$$
\mu: E_{T} \times N \rightarrow E_{T},
$$

which extends right multiplication of $T$ on $E_{T}$.

Proof. Given $n=(\gamma, g) \in N$ we first define $v_{n}:=\beta_{g} \circ \gamma$, where $\beta_{g}$ is the pull-back of $g: Z \rightarrow Z$ via the projection map $p: E_{T} \times_{g}^{T} T \rightarrow Z$. This is summarized by the diagram

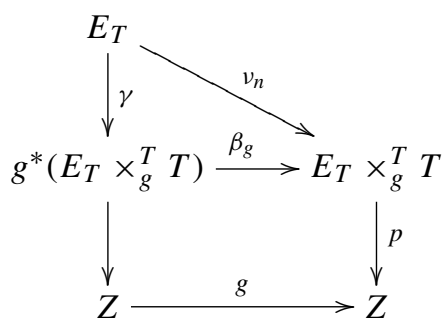

In order to define the morphism $\mu$, it suffices to define for any $n=(\gamma, g) \in N$ the right multiplication with $n$ on $E_{T}$,

$$
\mu_{n}: E_{T} \rightarrow E_{T} \quad \text { satisfying } \quad \mu_{n n^{\prime}}=\mu_{n^{\prime}} \circ \mu_{n} \forall n, n^{\prime} \in N .
$$

The set of morphisms $\left\{\mu_{n}\right\}_{n \in N}$ is related to the set of "twisted" morphisms $\left\{v_{n}\right\}_{n \in N}$ by composition

$$
\mu_{n}: E_{T} \stackrel{v_{n}}{\longrightarrow} E_{T} \times_{g}^{T} T \stackrel{\alpha_{g}}{\longrightarrow} E_{T} \quad \text { with } \quad \alpha_{g}[\overline{(e, t)}]=e g^{-1}(t) .
$$

Here $\overline{(e, t)}$ denotes the class of $(e, t) \in E_{T} \times T$ in the quotient $E_{T} \times{ }_{g}^{T} T:=E_{T} \times T / T$. Note that we use the equality etn $=e n g^{-1}(t)$ for $e \in E_{T}, n=(\gamma, g) \in N$ and $t \in T$.

It is now straightforward to check that the set $\left\{\mu_{n}\right\}_{n \in N}$ satisfies (13).

\subsection{The relative case}

In order to construct the abelianization map (see Section 7.3) we need to define the twisted Mumford group for families of $W$-invariant $T$-bundles. 
Let $S$ be a scheme and let $\mathcal{E}_{T}$ be a family of $T$-bundles over $Z$ parametrized by $S$, i.e. a $T$-bundle over the product $Z \times S$. Let $\pi_{S}: Z \times S \rightarrow S$ denote projection onto the second factor. We say that $\mathcal{E}_{T}$ is $W$-invariant if for any $g \in W$ and any closed point $s \in S$ there exists an isomorphism

$$
\mathcal{E}_{T \mid Z \times\{s\}} \simeq g . \mathcal{E}_{T \mid Z \times\{s\}} \cdot
$$

The following lemma is the analogue of the see-saw theorem for $T$-bundles (see $[\overline{\mathrm{Mu}}]$ ).

Lemma 6.4. Let $\mathcal{A}_{T}$ be a family of $T$-bundles over $Z$ parametrized by S. Suppose that for any closed point $s \in S$ the $T$-bundle $\mathcal{A}_{T \mid Z \times\{s\}}$ is trivial. Then there exists a $T$-bundle $M$ over $S$ such that $\mathcal{A}_{T} \simeq \pi_{S}^{*} M$.

Applying this lemma to 14 we see that for any $g \in W$ there exists a $T$-bundle $M_{g}$ over $S$ such that

$$
\mathcal{E}_{T} \simeq g \cdot \mathcal{E}_{T} \otimes \pi_{S}^{*} M_{g} .
$$

Moreover, the map $W \rightarrow H^{1}(S, \underline{T}), g \mapsto M_{g}$, is easily seen to be a group homomorphism.

We now define the $\sigma$-twisted Mumford group of the family $\mathcal{E}_{T}$ by

$$
\mathcal{G}^{\sigma}\left(\mathcal{E}_{T}\right):=\left\{(\gamma, g) \mid g \in W, \gamma: \mathcal{E}_{T} \rightarrow g \cdot \mathcal{E}_{T} \otimes \pi_{S}^{*} M_{g}\right\},
$$

where $\gamma$ is a $T$-equivariant isomorphism over $Z \times S$. We easily see that the relative Mumford group fits into the exact sequence

$$
1 \rightarrow \operatorname{Mor}(S, T) \rightarrow \mathcal{G}^{\sigma}\left(\mathcal{E}_{T}\right) \rightarrow W \rightarrow 1
$$

If $S$ is complete and connected, then $\operatorname{Mor}(S, T)=T$ and for any closed point $s \in S$ the restriction map

$$
\operatorname{res}_{S}: \mathcal{G}^{\sigma}\left(\mathcal{E}_{T}\right) \stackrel{\sim}{\longrightarrow} \mathcal{G}^{\sigma}\left(E_{T}\right), \quad(\gamma, g) \mapsto\left(\operatorname{res}_{S}(\gamma), g\right),
$$

is an isomorphism. Here $E_{T}:=\mathcal{E}_{T \mid Z \times\{s\}}$.

\subsection{Non-twisted Mumford groups}

Let $G$ be an affine algebraic group.

Definition 6.5. Given a principal $G$-bundle $E_{G}$ over $Z$ we define the (non-twisted) Mumford group of $E_{G}$ by

$$
\mathcal{G}^{1}\left(E_{G}\right):=\left\{(\gamma, g) \mid g \in W, \gamma: E_{G} \rightarrow g^{*} E_{G}\right\}
$$

where $\gamma$ is a G-equivariant isomorphism. The composition law in $\mathcal{G}^{1}\left(E_{G}\right)$ is given by

$$
(\gamma, g) \cdot\left(\mu, g^{\prime}\right)=\left(g^{*}(\mu) \circ \gamma, g g^{\prime}\right)
$$


As before, the group $\mathcal{G}^{1}\left(E_{G}\right)$ fits into the exact sequence

$$
1 \rightarrow \operatorname{Aut}\left(E_{G}\right) \rightarrow \mathcal{G}^{1}\left(E_{G}\right) \rightarrow W \rightarrow 1 .
$$

The induced action by conjugation of $W$ on $\operatorname{Aut}\left(E_{G}\right)$ is trivial.

Proposition 6.6. Let $E_{T}$ be a $W$-invariant $T$-bundle over $Z$ and set $N=\mathcal{G}^{\sigma}\left(E_{T}\right)$. Let $E_{N}:=E_{T} \times{ }^{T} N$ be the principal $N$-bundle obtained by extending the structure group from $T$ to $N$. Then the exact sequence (16) for $E_{N}$,

$$
1 \rightarrow \operatorname{Aut}\left(E_{N}\right) \rightarrow \mathcal{G}^{1}\left(E_{N}\right) \rightarrow W \rightarrow 1,
$$

splits canonically.

Proof. By Proposition 6.3 the variety $E_{T}$ admits a canonical $N$-action. Given $e \in E_{T}$ and $n=(\gamma, g) \in N$ we denote their product by $e n$. We consider the morphism

$$
\Phi_{n}: E_{T} \times{ }^{T} N \rightarrow E_{T} \times{ }^{T} N, \quad \Phi_{n}[\overline{(e, f)}]=\overline{\left(e n, n^{-1} f\right)},
$$

with $e \in E_{T}$ and $f \in N$. Then one easily checks that $\Phi_{n}$ is well-defined and that it makes the following diagram commute:

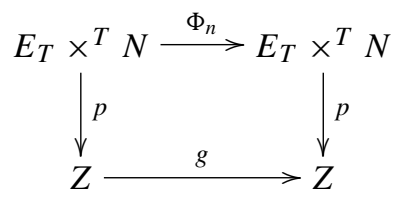

Hence $\Phi_{n}$ is a lift of the automorphism $g$ to the principal $N$-bundle $E_{T} \times{ }^{T} N$ and we may view $\Phi_{n}$ as an element of $\mathcal{G}^{1}\left(E_{N}\right)$. Moreover, $\Phi_{n n^{\prime}}=\Phi_{n^{\prime}} \circ \Phi_{n}$ and $\Phi_{t}=$ id for all $n, n^{\prime} \in N$ and all $t \in T$. Hence the morphism $\Phi_{n}$ only depends on $g \in W$. This gives the canonical splitting.

\subsection{Mumford groups and descent}

We recall the relation between the non-twisted Mumford group $\mathcal{G}^{1}\left(E_{G}\right)$ and descent.

Definition 6.7. Let $E_{G}$ be a $G$-bundle over $Z$. A $W$-linearization of the $G$-bundle $E_{G}$ is a splitting of the exact sequence (16),

$$
\mathcal{G}^{1}\left(E_{G}\right) \stackrel{\curvearrowleft}{\longmapsto} .
$$

Descent theory gives in the usual way (see [Gr2]

Proposition 6.8. Let $E_{G}$ be a $W$-linearized $G$-bundle over $Z$. There exists a $G$-bundle $F_{G}$ over $X$ such that $E_{G} \simeq \pi^{*} F_{G}$ if and only if for any ramification point $z \in Z$ of the covering $\pi: Z \rightarrow X$ the stabilizer subgroup $W_{z} \subset W$ of $z$ acts trivially on the fibre $\left(E_{G}\right)_{z}$.

Corollary 6.9. If the covering $\pi: Z \rightarrow X$ is étale, then given $E_{G}$ over $Z$ there is a bijection between the set of $W$-linearizations on $E_{G}$ and the set of $G$-bundles $F_{G}$ over $X$ such that $E_{G} \simeq \pi^{*} F_{G}$. 
Remark 6.10. We can rephrase Proposition 6.6 by saying that the $N$-bundle $E_{N}:=$ $E_{T} \times{ }^{T} N$ admits a canonical $W$-linearization. The conditions for descent of $E_{N}$ to $X$ given in Proposition 6.8 then translate into certain conditions on the $W$-invariant $T$-bundle $E_{T}$ (for the case of cameral coverings, see [DG]).

\subsection{The evaluation map $\mathrm{ev}_{\lambda}$ lifts}

Proposition 6.11. For any $\lambda \in \mathbb{S}_{\omega}$ the evaluation map $\mathrm{ev}_{\lambda}$ lifts to $\operatorname{Jac}(H ; Z)$, i.e. there is a commutative diagram

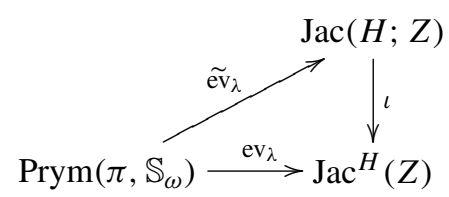

Proof. We have to show that the line bundle $L=\operatorname{ev}_{\lambda}\left(E_{T}\right)=E_{T} \times{ }_{\lambda}^{T} \mathbb{C}^{*}$ admits a canonical $H$-linearization. We denote by $\lambda=\lambda_{1}, \ldots, \lambda_{d} \in \mathbb{S}_{\omega}$ the $W$-orbit of the element $\lambda \in \mathbb{S}_{\omega}$, with $d=[W: H]$, and by $L_{i}:=E_{T} \times{ }_{\lambda_{i}}^{T} \mathbb{C}^{*} \in \operatorname{Jac}(Z)$ the line bundle obtained through $\lambda_{i}$ for $i=1, \ldots, d$. As before we set $N=\mathcal{G}^{\sigma}\left(E_{T}\right)$ and we consider $V=\operatorname{Ind}_{T}^{N}\left(\mathbb{C}_{\lambda}\right)$, the induced representation of $N$ from the representation $T \rightarrow \operatorname{GL}\left(\mathbb{C}_{\lambda}\right)$ given by $\lambda \in \operatorname{Hom}\left(T, \mathbb{C}^{*}\right)$. The restricted representation decomposes as a $T$-module $([\mathrm{Se}$. Proposition 15])

$$
\operatorname{Res}_{T}(V)=\left[\bigoplus_{i=1}^{d} \mathbb{C}_{\lambda_{i}}\right]^{\oplus|H|}
$$

which implies that the vector bundle $E_{N} \times{ }^{N} V$ decomposes as $\left[\bigoplus_{i=1}^{d} L_{i}\right]^{\oplus|H|}$. Moreover, by Proposition 6.6, $E_{N}$ admits a canonical $W$-linearization, hence the decomposable bundle $E_{N} \times{ }^{N} V$ also does. Since the subgroup $H \subset W$ preserves the direct summand $L=L_{1}$, we are done.

In Section 7.3 we will need the next lemma. First we introduce some notation. Given $\lambda \in \mathbb{S}_{\omega}$ we denote by $\lambda=\lambda_{1}, \ldots, \lambda_{d} \in \mathbb{S}_{\omega}$ the $W$-orbit of $\lambda$ and by $L_{i}:=E_{T} \times{ }_{\lambda_{i}}^{T} \mathbb{C}^{*}$ the associated line bundles for $i=1, \ldots, d$. We recall (see (10)) that if $\pi: Z \rightarrow X$ is étale, then $\operatorname{Jac}(Y)=\operatorname{Jac}(H ; Z)$, and we denote by $M \in \operatorname{Jac}(Y)$ the line bundle defined by the relation

$$
\varphi^{*} M=L_{1}=\operatorname{ev}_{\lambda}\left(E_{T}\right) .
$$

Lemma 6.12. The direct sum $\bigoplus_{i=1}^{d} L_{i}$ admits a canonical $W$-linearization, and if $\pi$ : $Z \rightarrow X$ is étale, then the $W$-linearized vector bundle $\bigoplus_{i=1}^{d} L_{i}$ equals $\pi^{*}\left(\psi_{*} M\right)$.

Proof. We have already seen in the proof of Proposition 6.11 that $\bigoplus_{i=1}^{d} L_{i}$ admits a canonical $W$-linearization. If $\pi: Z \rightarrow X$ is étale, then there exists a vector bundle $A$ over $X$ such that $\pi^{*} A=\bigoplus_{i=1}^{d} L_{i}$ as $W$-linearized vector bundles. By adjunction for the morphism $\psi: Y \rightarrow X$ we have $\operatorname{Hom}\left(A, \psi_{*} M\right)=\operatorname{Hom}\left(\psi^{*} A, M\right)$, and since $\pi$ is étale,

$$
\operatorname{Hom}\left(\psi^{*} A, M\right)=\operatorname{Hom}\left(\pi^{*} A, \varphi^{*} M\right)^{H}=\operatorname{Hom}\left(\bigoplus_{i=1}^{d} L_{i}, L_{1}\right)^{H},
$$


where the exponent $H$ denotes $H$-equivariant homomorphisms. The last space contains the projection onto the first factor, which is $H$-equivariant. This gives a non-zero homomorphism $\alpha: A \rightarrow \psi_{*} M$.

Similarly one can show using the isomorphism $\operatorname{Hom}\left(\psi_{*} M, \mathcal{O}_{X}\right)=\psi_{*}\left(M^{-1}\right)$ that $\operatorname{Hom}\left(\psi_{*} M, A\right)=\operatorname{Hom}\left(\bigoplus_{i=1}^{d} L_{i}^{-1}, L_{1}^{-1}\right)^{H}$. As before, projection onto the second factor gives a non-zero homomorphism $\beta: \psi_{*} M \rightarrow A$ and one easily checks that $\alpha \circ \beta=$ $\operatorname{id}_{\psi_{*} M}$. Hence $\alpha$ is an isomorphism and we are done.

\subsection{Relation between the Prym variety $P_{\lambda}$ and the Donagi-Prym $\operatorname{Prym}\left(\pi, \mathbb{S}_{\omega}\right)$}

By Proposition 6.11 there exists a morphism $\widetilde{e v}_{\lambda}: \operatorname{Prym}\left(\pi, \mathbb{S}_{\omega}\right) \rightarrow \operatorname{Jac}(H ; Z)$, and because of the exact sequence (10) the connected component $\operatorname{Prym}\left(\pi, \mathbb{S}_{\omega}\right)_{0}$ containing 0 is mapped by $\widetilde{\mathrm{ev}}_{\lambda}$ into $\mathrm{Jac}(Y)$.

Proposition 6.13. We have an equality of abelian subvarieties of $\operatorname{Jac}(Y)$,

$$
\tilde{\operatorname{ev}}_{\lambda}\left(\operatorname{Prym}\left(\pi, \mathbb{S}_{\omega}\right)_{0}\right)=P_{\lambda} \subset \operatorname{Jac}(Y) .
$$

Proof. We consider the natural map of $\mathbb{Z}$-algebras

$$
\phi: \bigoplus_{\omega \in \widehat{W}} \operatorname{End}\left(\mathbb{S}_{\omega}\right) \rightarrow \mathbb{Z}[W] .
$$

Note that $\phi \otimes_{\mathbb{Z}} \mathbb{Q}$ is an isomorphism of $\mathbb{Q}$-algebras $\bigoplus_{\omega \in \widehat{W}} \operatorname{End}\left(V_{\omega}\right) \cong \mathbb{Q}[W]$. Taking the tensor product over $\mathbb{Z}[W]$ with the $\mathbb{Z}[W]$-module $\operatorname{Jac}(Z)$ induces an isogeny decomposition of $\operatorname{Jac}(Z)$ (see also [Don1, formula (5.3)])

$$
\phi: \bigoplus_{\omega \in \widehat{W}} \mathbb{S}_{\omega} \otimes_{\mathbb{Z}} \operatorname{Prym}\left(\pi, \mathbb{S}_{\omega}\right) \rightarrow \operatorname{Jac}(Z) \otimes_{\mathbb{Z}[W]} \mathbb{Z}[W]=\operatorname{Jac}(Z) .
$$

Moreover, the restriction of $\phi$ to $\lambda \cdot \mathbb{Z} \otimes_{\mathbb{Z}} \operatorname{Prym}\left(\pi, \mathbb{S}_{\omega}\right)$ is the evaluation map ev $($ Section 5.1). With the notation of Lemma 3.8, multiplication with the idempotent $p_{\lambda}=(1 / e) S_{\lambda} \in$ $\mathbb{Q}[W]$ corresponds under $\phi \otimes_{Z} \mathbb{Q}$ to the projection onto the $\mathbb{Q}$-vector space $\lambda \cdot \mathbb{Q} \otimes_{\mathbb{Q}} V_{\omega}^{*} \subset$ $\bigoplus_{\omega \in \widehat{W}} \operatorname{End}\left(V_{\omega}\right)$ (see e.g. [Me, Section 4.4]). The proposition now follows immediately from the definition of the Prym variety $P_{\lambda}$.

\section{The abelianization map: the étale case}

\subsection{Grothendieck's spectral sequence}

We recall some facts relating to Grothendieck's spectral sequence [Gr1]. Let $W$ be any finite group (not necessarily a Weyl group), let $Z$ be a curve with a right $W$-action with quotient $\pi: Z \rightarrow X$, and let $A$ be an abelian algebraic group, which is also a $W$-module. Let $\underline{A}$ denote the $W$-sheaf of abelian groups defined by $\underline{A}(U)=\operatorname{Mor}(U, A)$ for an open subset $U \subset Z$. Consider the following two left-exact functors: 
$\Gamma_{Z}:\{W$-sheaves over $Z\} \rightarrow\{W$-modules $\}$,

$$
\Gamma_{Z}(\underline{A})=\Gamma(Z, \underline{A})=\text { global sections of } \underline{A},
$$

$\Gamma^{W}:\{W$-modules $\} \rightarrow$ abelian groups $\}$,

$$
\Gamma^{W}(M)=M^{W}=W \text {-invariant elements of } M \text {. }
$$

Consider the composite functor $\Gamma_{Z}^{W}=\Gamma^{W} \circ \Gamma_{Z}$. Its $n$-th derived functor, which we denote by $H^{n}(Z ; W ; \cdot)$, is computed by Grothendieck's spectral sequences

$$
\begin{gathered}
E_{2}^{p, q}=H^{p}\left(W, H^{q}(Z, \underline{A})\right) \Rightarrow E^{p+q}=H^{p+q}(Z ; W ; \underline{A}), \\
E_{2}^{p, q}=H^{p}\left(X, R^{q} \pi_{*}^{W}(\underline{A})\right) \Rightarrow E^{p+q}=H^{p+q}(Z ; W ; \underline{A}),
\end{gathered}
$$

and the associated exact sequence of low degree terms of the first spectral sequence is

$$
0 \rightarrow E_{2}^{1,0} \rightarrow E^{1} \rightarrow E_{2}^{0,1} \stackrel{c}{\longrightarrow} E_{2}^{2,0} \rightarrow E^{2} .
$$

In case $W$ acts on a torus $A=T$ with $\mathbb{S}_{\omega}=\operatorname{Hom}\left(T, \mathbb{C}^{*}\right)$ this exact sequence becomes, by Lemma 6.1 .

$$
0 \rightarrow H^{1}(W, T) \rightarrow H^{1}(Z ; W ; \underline{T}) \rightarrow \operatorname{Prym}\left(\pi, \mathbb{S}_{\omega}\right) \stackrel{c}{\longrightarrow} H^{2}(W, T) \rightarrow H^{2}(Z ; W ; \underline{T}) .
$$

One can work out the following description of the homomorphism $c$. Here we omit the details.

Proposition 7.1. For any $T$-bundle $E_{T} \in \operatorname{Prym}\left(\pi, \mathbb{S}_{\omega}\right)$ the cohomology class $c\left(E_{T}\right) \in$ $H^{2}(W, T)$ equals the extension class (12) of the twisted Mumford group $\mathcal{G}^{\sigma}\left(E_{T}\right)$.

Lemma 7.2. If the covering $\pi: Z \rightarrow X$ is étale, then $H^{2}(Z ; W ; \underline{T})=0$.

Proof. We use the second spectral sequence ${ }^{\prime} E_{2}^{p, q}$. First we observe that the sheaves $R^{1} \pi_{*}^{W}(\underline{T})$ and $R^{2} \pi_{*}^{W}(\underline{T})$ are supported on the ramification divisor of $\pi: Z \rightarrow X$ by [Gr1, Théorème 5.3.1]. Hence ' $E_{2}^{1,1}={ }^{\prime} E_{2}^{0,2}=0$.

Next we claim that $\pi^{*}\left(\pi_{*}^{W}(\underline{T})\right)=\underline{T}$. Let $V \subset Z$ be a sufficiently small open subset such that $g(V) \cap V=\varnothing$ for any $g \in W$ and $g \neq e$ (here we use the analytic topology on $Z$ ). Then the open subset $\pi^{-1}(\pi(V))$ decomposes as a disjoint union $\bigsqcup_{g \in W} g(V)$. Now the elements of $\pi^{*}\left(\pi_{*}^{W}(T)\right)(V)$ correspond to $W$-equivariant morphisms $\operatorname{Mor}^{W}\left(\pi^{-1}(\pi(V)), T\right)$. The restriction to $V$ gives a canonical bijection

$$
\operatorname{Mor}^{W}\left(\coprod_{g \in W} g(V), T\right)=\operatorname{Mor}(V, T)=\underline{T}(V),
$$

which proves the claim.

Finally, we observe that $\underline{T} \cong\left(\mathbb{C}^{*}\right)^{n}$ and since $H^{2}\left(Z, \underline{\mathbb{C}}^{*}\right)=0$ (this easily follows from the exponential exact sequence $0 \rightarrow \mathbb{Z} \rightarrow \mathcal{O}_{Z} \rightarrow \mathcal{O}_{Z}^{*} \rightarrow 0$ ), we see that $H^{2}(Z, \underline{T})=0$. We now conclude that ${ }^{\prime} E_{2}^{2,0}=H^{2}\left(X, \pi_{*}^{W}(\underline{T})\right)=0$, since $H^{2}\left(X, \pi_{*}^{W}(\underline{T})\right)$ identifies with the $W$-invariant subspace of $H^{2}(Z, \underline{T})$. 
For any $\lambda \in \mathbb{S}_{\omega}=\operatorname{Hom}\left(T, \mathbb{C}^{*}\right)$ let $H=\operatorname{Stab}(\lambda) \subset W$ and let $\beta_{\lambda}$ be the composite map

$$
\beta_{\lambda}: H^{2}(W, T) \stackrel{\operatorname{res}_{H}}{\longrightarrow} H^{2}(H, T) \stackrel{\lambda}{\longrightarrow} H^{2}\left(H, \mathbb{C}^{*}\right) .
$$

If the covering $\pi: Z \rightarrow X$ is not necessarily étale, we can say the following on the image of $c$. The next proposition will not be used as we will concentrate on the étale case.

Proposition 7.3. We have a commutative diagram

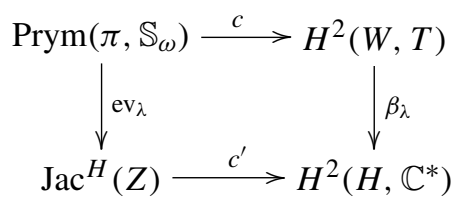

and $\beta_{\lambda} \circ c=0$ for any $\lambda \in \mathbb{S}_{\omega}$.

Proof. In order to prove that the diagram commutes it is sufficient to combine the three exact sequences $(17)$ obtained in the three cases $(W, T),(H, T)$ and $\left(H, \mathbb{C}^{*}\right)$; here the first factor denotes the finite group and the second the abelian algebraic group $A$ on which the finite group acts. We leave the details to the reader. The claim $\beta_{\lambda} \circ c=c^{\prime} \circ \mathrm{ev}_{\lambda}=0$ is an immediate consequence of Proposition 6.11 and (9).

\subsection{Moduli stack of G-bundles}

Let $G$ be a simple and simply connected algebraic group and let $T \subset G$ be a maximal torus, $N(T)$ the normalizer of the torus, and $W=N(T) / T$ the Weyl group of $G$. Let $\mathbb{S}_{\omega}:=\operatorname{Hom}\left(T, \mathbb{C}^{*}\right)$ be the weight lattice of $G$.

We denote by $\mathcal{M}_{X}(G)$ the moduli stack parametrizing the principal $G$-bundles over the curve $X$. We recall some results from [LS] and [So2] on line bundles over $\mathcal{M}_{X}(G)$. Let $\lambda \in \mathbb{S}_{\omega}$ be a dominant weight and $\rho_{\lambda}: G \rightarrow \operatorname{SL}\left(V_{\lambda}\right)$ the associated irreducible representation. Then $\rho_{\lambda}$ induces a morphism of stacks

$$
\tilde{\rho}_{\lambda}: \mathcal{M}_{X}(G) \rightarrow \mathcal{M}_{X}(\operatorname{SL}(m)), \quad E_{G} \mapsto E_{G} \times{ }^{G} V_{\lambda}
$$

Here $m=\operatorname{dim} V_{\lambda}$. We denote by $\mathcal{D}_{\lambda}=\widetilde{\rho}_{\lambda}^{*} \mathcal{D}$ the pull-back of the determinant line bundle $\mathcal{D}$ over $\mathcal{M}_{X}(\mathrm{SL}(m))$. The next proposition is proved in [LS] and [So2].

Proposition 7.4. For any simple and simply connected algebraic group $G$ we have:

(1) There exists an ample line bundle $\mathcal{L}$ over $\mathcal{M}_{X}(G)$ such that $\operatorname{Pic}\left(\mathcal{M}_{X}(G)\right) \cong \mathbb{Z} \cdot \mathcal{L}$.

(2) For any dominant weight $\lambda \in \mathbb{S}_{\omega}$, the integer $d_{\lambda}$ defined by the relation

$$
\mathcal{D}_{\lambda}=\mathcal{L}^{\otimes d_{\lambda}}
$$

equals the Dynkin index of the representation $\rho_{\lambda}$ of $G$. 


\subsection{The abelianization map $\Delta_{\theta}$}

From now on we assume that the Galois covering $\pi: Z \rightarrow X$ is étale with Galois group equal to the Weyl group $W=N(T) / T$. We denote by $n \in H^{2}(W, T)$ the extension class of $N(T)$. Note that by Lemma 7.2 and (18) the homomorphism

$$
c: \operatorname{Prym}\left(\pi, \mathbb{S}_{\omega}\right) \rightarrow H^{2}(W, T)
$$

is surjective. We denote by $\operatorname{Prym}\left(\pi, \mathbb{S}_{\omega}\right)_{n}$ a connected component of the fibre of $c$ over $n$. Note that all connected components are isomorphic to each other.

As in Section 6.2, we consider a universal family $\mathcal{E}_{T}$ of $T$-bundles over $Z \times$ $\operatorname{Prym}\left(\pi, \mathbb{S}_{\omega}\right)_{n}$ (note that $\mathcal{E}_{T}$ is $W$-invariant) and we choose an isomorphism

$$
\theta: \mathcal{G}^{\sigma}\left(\mathcal{E}_{T}\right) \stackrel{\sim}{\longrightarrow} N(T)
$$

inducing the identity on the subgroups $T$ of $\mathcal{G}^{\sigma}\left(\mathcal{E}_{T}\right)$ and $N(T)$ and on the quotient $W$. The existence of $\theta$ follows from $\left[15\right.$ and the fact that $\operatorname{Prym}\left(\pi, \mathbb{S}_{\omega}\right)_{n}$ lies in the fibre of $c$ over $n$, the extension class of $N(T)$.

Proposition 7.5. Given an isomorphism $\theta$ there exists a morphism

$$
\Delta_{\theta}: \operatorname{Prym}\left(\pi, \mathbb{S}_{\omega}\right)_{n} \rightarrow \mathcal{M}_{X}(G)
$$

such that for any $E_{T} \in \operatorname{Prym}\left(\pi, \mathbb{S}_{\omega}\right)_{n}$ the $G$-bundle $\Delta_{\theta}\left(E_{T}\right)$ satisfies

$$
\pi^{*} \Delta_{\theta}\left(E_{T}\right)=E_{T} \times^{T} G .
$$

The map $\Delta_{\theta}$ is called the abelianization map.

Proof. The existence of the morphism $\Delta_{\theta}$ will follow from the existence of a family of $N(T)$-bundles over $X$ parametrized by $\operatorname{Prym}\left(\pi, \mathbb{S}_{\omega}\right)_{n}$, or equivalently from the existence of a family of $N(T)$-bundles over $Z$ with a $W$-linearization parametrized by $\operatorname{Prym}\left(\pi, \mathbb{S}_{\omega}\right)_{n}$. But this follows from the relative version of Proposition 6.6, which says that the $N(T)$-bundle $\mathcal{E}_{T} \times{ }_{\theta}^{T} N(T)$ admits a canonical $W$-linearization; here we use the isomorphism $\theta$.

Remark 7.6. (i) Note that $\Delta_{\theta}$ factorizes through $\mathcal{M}_{X}(N(T))$.

(ii) A priori $\Delta_{\theta}$ depends on the choice of $\theta$. Two different choices of $\theta$ differ by an element in $\operatorname{Aut}^{0}(N(T))$, the group of automorphisms of $N(T)$ inducing the identity on $T$ and $W$. Note that $T \subset \operatorname{Aut}^{0}(N(T))$ and $\operatorname{Aut}^{0}(N(T)) / T=H^{1}(W, T)$. The cohomology groups $H^{1}(W, T)$ and $H^{2}(W, T)$ have been computed in [Mal.

\subsection{Direct images of line bundles}

Consider a dominant weight $\lambda \in \mathbb{S}_{\omega}$ and let $V_{\lambda}$ denote the associated irreducible representation of $G$. We consider the weight space decomposition

$$
V_{\lambda}=\bigoplus_{\mu \in \Theta(\lambda)} V_{\lambda}^{\mu}
$$


where $V_{\lambda}^{\mu}$ denotes the weight space of $V_{\lambda}$ associated to the weight $\mu \in \mathbb{S}_{\omega}$. The Weyl group $W$ acts on the set $\Theta(\lambda)$ of all weights $\mu$ such that $V_{\lambda}^{\mu} \neq\{0\}$ and decomposes it into $k$ orbit spaces

$$
\Theta(\lambda)=\Theta_{1} \cup \cdots \cup \Theta_{k} .
$$

For each $j=1, \ldots, k$ we choose a weight $\lambda_{j} \in \Theta_{j}$, i.e. $\Theta_{j}=\lambda_{j} . W$. We introduce for each $j$ the subgroup $H_{j}=\operatorname{Stab}\left(\lambda_{j}\right) \subset W$ and the quotient $\psi_{j}: Z / H_{j}=Y_{j} \rightarrow X$. Let $n_{j}=\operatorname{dim} V_{\lambda}^{\lambda_{j}}$. Note that $n_{j}=\operatorname{dim} V_{\lambda}^{\mu}$ for any $\mu \in \Theta_{j}$, and $\lambda_{1}=\lambda$ and $n_{1}=1$.

Since $\pi$ is étale, we have $\operatorname{Jac}\left(H_{j} ; Z\right)=\operatorname{Jac}\left(Y_{j}\right)$ and by Proposition 6.11 there exist morphisms

$$
\widetilde{\operatorname{ev}}_{\lambda_{j}}: \operatorname{Prym}\left(\pi, \mathbb{S}_{\omega}\right) \rightarrow \operatorname{Jac}\left(Y_{j}\right) .
$$

For convenience of notation we introduce the product and the map

$$
\operatorname{Jac}\left(Y_{\bullet}\right):=\operatorname{Jac}\left(Y_{1}\right) \times \cdots \times \operatorname{Jac}\left(Y_{k}\right), \quad \widetilde{\mathrm{ev}}_{\bullet}:=\left(\widetilde{\mathrm{ev}}_{\lambda_{1}}, \ldots, \widetilde{\mathrm{ev}}_{\lambda_{k}}\right),
$$

and the direct image morphism

$$
\psi_{\bullet}: \operatorname{Jac}\left(Y_{\bullet}\right) \rightarrow \mathcal{M}_{X}(\mathrm{GL}(m)), \quad\left(M_{1}, \ldots, M_{k}\right) \mapsto \bigoplus_{j=1}^{k}\left[\psi_{j *}\left(M_{j}\right)\right]^{\oplus n_{j}} .
$$

Proposition 7.7. Asume $\pi$ is étale. Then we have a commutative diagram

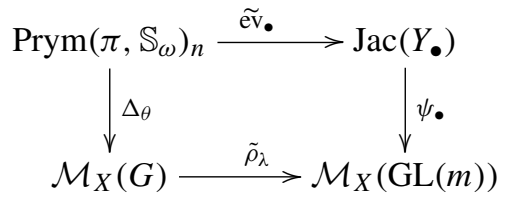

Proof. Let $E_{T} \in \operatorname{Prym}\left(\pi, \mathbb{S}_{\omega}\right)_{n}$. The rank- $m$ vector bundle $\tilde{\rho}_{\lambda}\left[\Delta_{\theta}\left(E_{T}\right)\right]$ pulls back under $\pi$ to the decomposable $W$-linearized vector bundle over $Z$,

$$
E_{T} \times{ }^{T} V_{\lambda}=\bigoplus_{\mu \in \Theta(\lambda)} E_{T} \times{ }^{T} V_{\lambda}^{\mu}=\bigoplus_{j=1}^{k}\left[\bigoplus_{\mu \in \Theta_{j}} E_{T} \times{ }_{\mu}^{T} \mathbb{C}\right]^{\oplus n_{j}} .
$$

Clearly the $W$-linearization preserves the direct summands $\bigoplus_{\mu \in \Theta_{j}} E_{T} \times_{\mu}^{T} \mathbb{C}$ for $j=$ $1, \ldots, k$ and by Lemma 6.12 we have the equality

$$
\bigoplus_{\mu \in \Theta_{j}} E_{T} \times{ }_{\mu}^{T} \mathbb{C}=\pi^{*}\left(\psi_{j *}\left(M_{j}\right)\right)
$$

as $W$-linearized vector bundles. Here $M_{j}=\widetilde{\mathrm{ev}}_{j}\left(E_{T}\right)$. This proves the claim.

Remark 7.8. Note that the composite map $\psi_{\bullet} \circ \widetilde{\mathrm{ev}}_{\bullet}=\tilde{\rho}_{\lambda} \circ \Delta_{\theta}$ takes values in $\mathcal{M}_{X}(\operatorname{SL}(m))$.

\section{Proof of the main theorem}

For the convenience of the reader we recall the set-up of our main result. Let $G$ be a simple and simply connected algebraic group and $T \subset G$ a maximal torus. Let $W:=N(T) / T$ denote the Weyl group of $G$ and $\mathbb{S}_{\omega}:=\operatorname{Hom}\left(T, \mathbb{C}^{*}\right)$ the weight lattice. Let $\pi: Z \rightarrow X$ 
be an étale Galois covering of smooth projective curves with Galois group $W$. For a dominant weight $\lambda \in \mathbb{S}_{\omega}$ we consider the Prym variety (see Section 3.5) $P_{\lambda} \subset \operatorname{Jac}(Y)$ with $Y=Z / H$ and $H=\operatorname{Stab}(\lambda)$. Let $\bar{K}_{\lambda}$ denote the Kanev correspondence on the curve $Y$. Let $L_{Y}$ be a line bundle over $\operatorname{Jac}(Y)$ representing the principal polarization. Note that $L_{Y}$ is uniquely defined up to translation. Then we can prove our main result.

Theorem 8.1. Assume that:

- the $W$-covering $\pi: Z \rightarrow X$ is étale,

- $q_{\lambda}=d_{\lambda}$,

- the group $\Gamma_{\lambda}=\mathbb{S}_{\omega} / \lambda \cdot \mathbb{Z}[W]$ is trivial,

- the weight $\lambda$ is minuscule or quasi-minuscule,

- the homomorphism $\psi^{*}: \operatorname{Jac}(X) \rightarrow \operatorname{Jac}(Y)$ is injective.

Then

(1) there exists a line bundle $M$ over $P_{\lambda}$ such that $L_{Y} \mid P_{\lambda}=M^{\otimes q_{\lambda}}$,

(2) the type of the polarization $M$ over $P_{\lambda}$ equals

$$
K(M)=(\mathbb{Z} / m \mathbb{Z})^{2 g} \quad \text { with } \quad m=\frac{\operatorname{deg}(Y / X)}{\operatorname{gcd}\left(\operatorname{deg}\left(\bar{K}_{\lambda}\right)-1, \operatorname{deg}(Y / X)\right)},
$$

and $g$ denotes the genus of $X$.

Proof. (1) If $\pi$ is étale, Proposition 6.11 gives a morphism

$$
\widetilde{\operatorname{ev}}_{\lambda}: \operatorname{Prym}\left(\pi, \mathbb{S}_{\omega}\right) \rightarrow \operatorname{Jac}(H ; Z)=\operatorname{Jac}(Y) .
$$

Moreover, by Proposition 6.13 we have $\widetilde{e v}_{\lambda}\left(\operatorname{Prym}\left(\pi, \mathbb{S}_{\omega}\right)_{0}\right)=P_{\lambda}$. Since we have assumed that $\Gamma_{\lambda}$ is trivial, the evaluation map ev $v_{\lambda}$ is injective by Proposition 5.2. Hence the map $\widetilde{\mathrm{ev}}_{\lambda}$ is also injective and induces isomorphisms by restriction

$$
\widetilde{\operatorname{ev}}_{\lambda}: \operatorname{Prym}\left(\pi, \mathbb{S}_{\omega}\right)_{0} \stackrel{\sim}{\longrightarrow} P_{\lambda} \subset \operatorname{Jac}(Y), \quad \widetilde{\operatorname{ev}}_{\lambda}: \operatorname{Prym}\left(\pi, \mathbb{S}_{\omega}\right)_{n} \stackrel{\sim}{\longrightarrow} T_{\alpha}\left(P_{\lambda}\right) \subset \operatorname{Jac}(Y),
$$

where $T_{\alpha}\left(P_{\lambda}\right)$ denotes the translate by an element $\alpha \in \operatorname{Jac}(Y)$ of the Prym variety $P_{\lambda}$. In order to show (1) it suffices to show that $L_{Y} \mid T_{\alpha}\left(P_{\lambda}\right)$ is divisible by $q_{\lambda}$.

We first consider the case of $\lambda$ minuscule, i.e. $k=1$ in the notation of Section 7.4. In that case the commutative diagram of Proposition 7.7 simplifies to

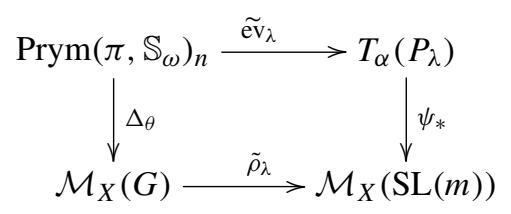

In the notation of Section 7.2 we know that $\tilde{\rho}_{\lambda}^{*} \mathcal{D}=\mathcal{L}^{\otimes d_{\lambda}}$. Hence the line bundle

$$
\widetilde{\mathrm{ev}}_{\lambda}^{*}\left(\left(\psi_{*}\right)^{*} \mathcal{D}\right)=\Delta_{\theta}^{*}\left(\tilde{\rho}_{\lambda}^{*} \mathcal{D}\right)=\left(\Delta_{\theta}^{*} \mathcal{L}\right)^{\otimes d_{\lambda}}
$$


is divisible by $d_{\lambda}=q_{\lambda}$. Since the first horizontal map $\tilde{\mathrm{ev}}_{\lambda}$ is an isomorphism, we deduce that $\left(\psi_{*}\right)^{*} \mathcal{D}=L_{Y} \mid T_{\alpha}\left(P_{\lambda}\right)$ is also divisible by $q_{\lambda}$.

In case $\lambda$ is quasi-minuscule, we have $k=2$ and $\lambda_{2}=0$. Hence $H_{2}=\operatorname{Stab}(0)=W$ and $\psi_{2}=$ id $: Y_{2}=X \rightarrow X$. Moreover, $\widetilde{e v}_{0}: \operatorname{Prym}\left(\pi, \mathbb{S}_{\omega}\right)_{n} \rightarrow \operatorname{Jac}(X)$ is the constant zero map and the commutative diagram of Proposition 7.7 simplifies to

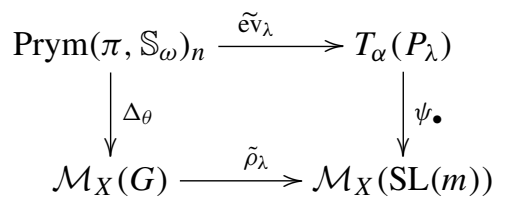

with $\psi_{\bullet}(M)=\psi_{1 *}(M) \oplus \mathcal{O}_{X}^{\oplus n_{2}}$ for $M \in \operatorname{Jac}(Y)$. We then conclude as in the case of a minuscule weight.

(2) Let $S$ denote the usual Prym variety $\operatorname{Prym}(Y / X)$. Then we recall from Section 3.5 that $P_{\lambda}=\operatorname{im}\left(u_{\lambda}\right) \subset S$ and that the endomorphism $u_{\lambda} \in \operatorname{End}(S)$ satisfies the relation $u_{\lambda}^{2}=q_{\lambda} u_{\lambda}$. Hence we can apply Proposition 2.10 (a), which says that the type of the polarization $K(M)$ is given by

$$
K(M)=u_{\lambda}\left(K\left(L_{Y} \mid S\right)\right),
$$

where $L_{Y} \mid S$ denotes the restriction of the line bundle $L_{Y}$ to $S$. Since $\psi^{*}: \operatorname{Jac}(X) \rightarrow$ $\operatorname{Jac}(Y)$ is injective, we have (see Remark 2.1(ii)) the equalities $S=\operatorname{Prym}(Y / X)=$ $\operatorname{im}(d-t)$ and $\psi^{*} \operatorname{Jac}(X)=\operatorname{im} t$, where $t \in \operatorname{End}(\operatorname{Jac}(Y))$ denotes the endomorphism associated to the trace correspondence of $\psi: Y \rightarrow X$. By [BL, Corollary 12.1.4] or Lemma 2.5 we obtain

$$
K\left(L_{Y} \mid S\right)=K\left(L_{Y} \mid \operatorname{Jac}(X)\right)=\operatorname{Jac}(X) \cap S .
$$

Moreover, $K\left(L_{Y} \mid \operatorname{Jac}(X)\right)=\psi^{*}\left[\operatorname{Jac}(X)_{d}\right] \cong(\mathbb{Z} / d \mathbb{Z})^{2 g}$, with $d=\operatorname{deg}(Y / X)$. We deduce from Corollary 3.7 2 ) that $u_{\lambda} t=\left(\operatorname{deg}\left(\bar{K}_{\lambda}\right)-1\right) t$, and since $K\left(L_{Y} \mid S\right) \subset$ $\operatorname{im} t=\psi^{*} \operatorname{Jac}(X)$ we conclude that $u_{\lambda}\left(K\left(L_{Y} \mid S\right)\right)$ equals the image of multiplication by $\operatorname{deg}\left(\bar{K}_{\lambda}\right)-1$ in the group $(\mathbb{Z} / d \mathbb{Z})^{2 g}$. This proves (2).

We deduce from Lemma 5.4 and from the two tables of Section 4 the following list of examples satisfying the five conditions of Theorem 8.1 This shows the main theorem stated in the introduction.

Corollary 8.2. The type $K(M)=(\mathbb{Z} / m \mathbb{Z})^{2 g}$ of the induced polarization $L_{Y \mid P_{\lambda}}=M^{\otimes q_{\lambda}}$ on the Prym variety $P_{\lambda}$ is given by the table below.

\begin{tabular}{cccc}
\hline Weyl group of type & weight & $q_{\lambda}=d_{\lambda}$ & $m$ \\
\hline$A_{n}, n>1$ & $\varpi_{i},(i, n+1)=1$ & $\left(\begin{array}{c}n-1 \\
i-1\end{array}\right)$ & $n+1$ \\
$D_{n}, n$ odd & $\varpi_{n-1}, \varpi_{n}$ & $2^{n-3}$ & 4 \\
$E_{6}$ & $\varpi_{1}, \varpi_{6}$ & 6 & 3 \\
$E_{7}$ & $\varpi_{7}$ & 12 & 2 \\
$E_{8}$ & $\varpi_{8}$ & 60 & 1 \\
\hline
\end{tabular}


Proof. We only have to check that $\psi^{*}: \operatorname{Jac}(X) \rightarrow \operatorname{Jac}(Y)$ is injective in the cases mentioned in the table. This follows from the formula $\operatorname{ker} \psi^{*}=\operatorname{ker}\left(W^{*} \rightarrow H^{*}\right)$, where $W^{*}$ and $H^{*}$ denote the groups of characters of $W$ and $H$, and from a straightforward case-bycase study.

Remark 8.3. Under the assumption of the Main Theorem, we can identify $\operatorname{Prym}\left(\pi, \mathbb{S}_{\omega}\right)_{n}$ and $P_{\lambda}$ by composing the isomorphism $\tilde{\mathrm{ev}}_{\lambda}: \operatorname{Prym}\left(\pi, \mathbb{S}_{\omega}\right)_{n} \stackrel{\sim}{\longrightarrow} T_{\alpha}\left(P_{\lambda}\right)$ and the translation $T_{-\alpha}: T_{\alpha}\left(P_{\lambda}\right) \stackrel{\sim}{\longrightarrow} P_{\lambda}$. Via this identification, the abelianization map $\Delta_{\theta}$ defined in Proposition 7.5 gives a morphism

$$
\gamma: P_{\lambda} \rightarrow \mathcal{M}_{X}(G)
$$

Moreover, by the proof of Theorem 8.1(1), the line bundle $\gamma^{*} \mathcal{L}$ is algebraically equivalent to $M$. Therefore choosing a suitable translate $L_{Y}$ representing the principal polarization on $\operatorname{Jac}(Y)$, we can assume that $M=\gamma^{*} \mathcal{L}$, i.e., $L_{Y} \mid P_{\lambda}=\left(\gamma^{*} \mathcal{L}\right)^{\otimes q_{\lambda}}$.

Remark 8.4. As mentioned in the introduction, it is natural to ask whether the linear map

$$
\gamma^{*}: H^{0}\left(\mathcal{M}_{X}(G), \mathcal{L}\right) \rightarrow H^{0}\left(P_{\lambda}, M\right)
$$

is an isomorphism.

In the case $G=\operatorname{SL}(m)$ we can answer this question affirmatively. Both vector spaces carry a natural linear action of the Heisenberg group, a central extension of the group of $m$-torsion points $\operatorname{Jac}(X)_{m}$, and are irreducible representations of this group. Therefore it suffices to show that $\gamma^{*}$ is non-zero, which is achieved by computing the pull-back $\gamma^{*}\left(\Theta_{N}\right)$ of the generalized theta divisor $\Theta_{N} \subset \mathcal{M}_{X}(\operatorname{SL}(m))$ with $N \in \operatorname{Pic}^{g-1}(X)$ (see $[\overline{\mathrm{BNR}}])$. Moreover, the theta divisor on $\operatorname{Jac}(Y)$ induces by pull-back to $P_{\lambda} \times \operatorname{Jac}(X)$ an isomorphism

$$
H^{0}\left(P_{\lambda}, M\right) \stackrel{\sim}{\longrightarrow} H^{0}\left(\operatorname{Jac}(X), m L_{X}\right)^{*},
$$

where $L_{X}$ represents the principal polarization on $\operatorname{Jac}(X)$ (see e.g. [BL] Section 12.1]).

In the remaining cases we do not know the answer, mainly because we are lacking a description of special divisors in $H^{0}\left(\mathcal{M}_{X}(G), \mathcal{L}\right)$. Especially intriguing is the case $G=E_{8}$, where both spaces are one-dimensional (see also [So2]). A description of $H^{0}\left(\mathcal{M}_{X}(G), \mathcal{L}\right)$ in terms of theta functions on $\operatorname{Jac}(X)$ as for $G=\operatorname{SL}(m)$ seems to be unavailable, since there are residual components to $P_{\lambda}$ and $\operatorname{Jac}(X)$ in the isogeny decomposition of $\operatorname{Jac}(Y)$ (see [Don1, formula (6.6)]).

Acknowledgments. We would like to thank Laurent Manivel for a helpful discussion.

\section{References}

[BNR] Beauville, A., Narasimhan, M. S., Ramanan, S.: Spectral covers and the generalised theta divisor. J. Reine Angew. Math. 398, 169-179 (1989) Zbl 0666.14015 MR 0998478

[BL] Birkenhake, Ch., Lange, H.: Complex Abelian Varieties. 2nd ed., Grundlehren Math. Wiss. 302, Springer (2004) Zbl 1056.14063 MR 2062673 
[Bo] Bourbaki, N.: Groupes et algèbres de Lie, Chapitres 4, 5 et 6. Hermann (1968) Zbl 0483.22001 MR 0240238

[Dol] Dolgachev, I.: Invariant stable bundles over modular curves $X(p)$. In: Recent Progress in Algebra (Taejon/Seoul, 1997), Contemp. Math. 224, Amer. Math. Soc., Providence, RI, 65-99 (1999) Zbl 0977.14012 MR 1653063

[Don1] Donagi, R.: Decomposition of spectral covers. In: Journées de géométrie algébrique d'Orsay, Astérisque 218, 145-176 (1993) Zbl 0820.14031 MR 1265312

[Don2] Donagi, R.: Spectral covers. In: Current Topics in Complex Algebraic Geometry (Berkeley, CA, 1992/93), Cambridge Univ. Press, Cambridge, 65-86 (1995) Zbl 0877.14026 MR 1397059

[DG] Donagi, R., Gaitsgory, D.: The gerbe of Higgs bundles, Transform. Groups 7, 109-153 (2002) Zbl 1083.14519 MR 1903115

[Fa1] Faltings, G.: A proof for the Verlinde formula. J. Algebraic Geom. 3, 347-374 (1994) Zbl 0809.14009 MR 1257326

[Fa2] Faltings, G.: Stable $G$-bundles and projective connections. J. Algebraic Geom. 2, 507-568 (1993) Zbl 0790.14019 MR 1211997

[Fu] Fulton, W.: Intersection Theory. Ergeb. Math. Grenzgeb. 2, Springer (1984) Zbl 0541.14005 MR 0732620

[Gr1] Grothendieck, A.: Sur quelques points d'algèbre homologique. Tohoku Math. J. (2) 9, 119221 (1957) Zbl 0118.26104 MR 0102537

[Gr2] Grothendieck, A.: Technique de descente et théorèmes d'existence en géométrie algébrique. Séminaire Bourbaki, exp. 190 (1959) Zbl 0229.14007 MR 1603475

[Ha] Hall, M.: Combinatorial Theory. 2nd ed., Wiley (1986). Zbl 0588.05001 MR 1635901

[Hi1] Hitchin, N.: The self-duality equations on a Riemann surface. Proc. London Math. Soc. (3) 55, 59-126 (1987) Zbl 0634.53045 MR 0887284

[Hi2] Hitchin, N.: Stable bundles and integrable systems. Duke Math. J. 54, 91-114 (1987) Zbl 0627.14024 MR 0885778

[K1] Kanev, V.: Spectral curves and Prym-Tjurin varieties I. In: Abelian Varieties (Egloffstein, 1993), de Gruyter, 151-198 (1995) Zbl 0856.14010 MR 1336606

[K2] Kanev, V.: Principal polarizations of Prym-Tyurin varieties. Compos. Math. 64, 243-270 (1987) Zbl 0694.14009 MR 0918413

[LR] Lange, H., Recillas, S.: Abelian varieties with group action. J. Reine Angew. Math. 575, 135-155 (2004) Zbl 1072.14053 MR 2097550

[LRo] Lange, H., Rojas, A.: A Galois theoretic approach to Kanev's correspondence. Manuscripta Mathematica 125, 225-240 (2008)

[LS] Laszlo, Y., Sorger, C.: The line bundles on the moduli of parabolic $G$-bundles over curves and their sections. Ann. Sci. École Norm. Sup. (4) 30, 499-525 (1997) Zbl 0918.14004 MR 1456243

[Ma] Matthey, M.: Normalizers of maximal tori and cohomology of Weyl groups. Preprint, http://igat.epfl.ch/matthey/publications/doc/weylcoho.pdf

[Me] Mérindol, J.-Y.: Variétés de Prym d'un revêtement galoisien. J. Reine Agew. Math. 461, 49-61 (1995) Zbl 0814.14043 MR 1324208

[Mu Mumford, D.: Abelian Varieties. Tata Inst. Fund. Res. Stud. Math. 5, Bombay, Oxford Univ. Press, London (1970) Zbl 0223.14022 MR 0282985

[O] Oxbury, W.: Spin Verlinde spaces and Prym theta functions. Proc. London Math. Soc. (3) 78, 52-76 (1999) Zbl 1031.14024 MR 1658152

[Se] Serre, J.-P.: Représentations linéaires des groupes finis. Hermann, Paris (1967) Zbl 0189.02603 MR 0232867 
[So1] Sorger, C.: La formule de Verlinde. In: Sém. Bourbaki, 1994/95, exp. 794, Astérisque 237, 87-114 (1996) Zbl 0878.17024 MR 1423621

[So2] Sorger, C.: On moduli of $G$-bundles of a curve for exceptional $G$. Ann. Sci. École Norm. Sup. (4) 32, 127-133 (1999) Zbl 0969.14016 MR 1670528

[Sp] Springer, T. A.: A construction of representations of Weyl groups. Invent. Math. 44, 279293 (1978) Zbl 0376.17002 MR 0491988 